\title{
Maresin 1 Promotes Inflammatory Resolution, Neuroprotection, and Functional Neurological Recovery After Spinal Cord Injury
}

\author{
Isaac Francos-Quijorna, ${ }^{1}$ Eva Santos-Nogueira, ${ }^{1}$ Karsten Gronert, ${ }^{2}$ Aaron B. Sullivan, ${ }^{2}{ }^{\odot}$ Marcel A. Kopp ${ }^{3}$ \\ (DBenedikt Brommer, ${ }^{3,4}$ Samuel David, ${ }^{-}{ }^{-}$Jan M. Schwab, ${ }^{3,6,7}$ and ${ }^{\circledR}$ Ruben López-Vales ${ }^{1}$ \\ ${ }^{1}$ Departament de Biologia Cel-lular, Fisiologia i Immunologia, Institut de Neurociencies, Centro de Investigacio'n Biome'dica en Red sobre Enfermedades \\ Neurodegenerativas (CIBERNED), Universitat Autonoma de Barcelona, 08193 Bellaterra, Catalonia, Spain, ${ }^{2}$ Vision Science Program, School of Optometry, \\ University of California Berkeley, Berkeley, California 94598, ${ }^{3}$ Spinal Cord Alliance Berlin (SCAB) and Department of Neurology and Experimental \\ Neurology, Charité Campus Mitte, Clinical and Experimental Spinal Cord Injury Research Laboratory (Neuroparaplegiology), Charité-Universitätsmedizin \\ Berlin, 10117 Berlin, Germany, ${ }^{4}$ F.M. Kirby Neurobiology Center, Boston Children's Hospital, and Department of Neurology, Harvard Medical School, \\ Boston, Massachusetts 02115, ${ }^{5}$ Centre for Research in Neuroscience, The Research Institute of the McGill University Health Centre, H3G1A4 Montreal, \\ Canada, and ${ }^{6}$ Department of Neurology, Spinal Cord Injury Division, and ${ }^{7}$ Department of Neuroscience and Center for Brain and Spinal Cord Repair, \\ Department of Physical Medicine and Rehabilitation, The Neurological Institute, The Ohio State University, Wexner Medical Centre, Columbus, Ohio 43210
}

Resolution of inflammation is defective after spinal cord injury (SCI), which impairs tissue integrity and remodeling and leads to functional deficits. Effective pharmacological treatments for SCI are not currently available. Maresin 1 (MaR1) is a highly conserved specialized proresolving mediator (SPM) hosting potent anti-inflammatory and proresolving properties with potent tissue regenerative actions. Here, we provide evidence that the inappropriate biosynthesis of SPM in the lesioned spinal cord hampers the resolution of inflammation and leads to deleterious consequences on neurological outcome in adult female mice. We report that, after spinal cord contusion injury in adult female mice, the biosynthesis of SPM is not induced in the lesion site up to 2 weeks after injury. Exogenous administration of MaR1, a highly conserved SPM, propagated inflammatory resolution after SCI, as revealed by accelerated clearance of neutrophils and a reduction in macrophage accumulation at the lesion site. In the search of mechanisms underlying the proresolving actions of MaR1 in SCI, we found that this SPM facilitated several hallmarks of resolution of inflammation, including reduction of proinflammatory cytokines (CXCL1, CXCL2, CCL3, CCL4, IL6, and CSF3), silencing of major inflammatory intracellular signaling cascades (STAT1, STAT3, STAT5, p38, and ERK1/2), redirection of macrophage activation toward a prorepair phenotype, and increase of the phagocytic engulfment of neutrophils by macrophages. Interestingly, MaR1 administration improved locomotor recovery significantly and mitigated secondary injury progression in a clinical relevant model of SCI. These findings suggest that proresolution, immunoresolvent therapies constitute a novel approach to improving neurological recovery after acute SCI.

Key words: lipid mediators; Maresin-1; neuroprotection; resolution; spinal cord injury

Significance Statement

Inflammation is a protective response to injury or infection. To result in tissue homeostasis, inflammation has to resolve over time. Incomplete or delayed resolution leads to detrimental effects, including propagated tissue damage and impaired wound healing, as occurs after spinal cord injury (SCI). We report that inflammation after SCI is dysregulated in part due to inappropriate synthesis of proresolving lipid mediators. We demonstrate that the administration of the resolution agonist referred to as maresin 1 (MaR1) after SCI actively propagates resolution processes at the lesion site and improves neurological outcome. MaR1 is identified as an interventional candidate to attenuate dysregulated lesional inflammation and to restore functional recovery after SCI. 


\section{Introduction}

Spinal cord injury (SCI) causes an immune response (David et al., 2012a; Gomez-Nicola and Perry, 2015; Steinman, 2015) composed of activated resident glial cells (microglia and astrocytes) and blood-derived leukocytes (neutrophils, monocytes, and lymphocytes) that enter the damaged spinal cord (Hawthorne and Popovich, 2011; Prüss et al., 2011; David et al., 2012a). These immune cells are required for effective clearance of damaged cell and myelin debris and for the release of bioactive molecules that lead to tissue healing and repair (Popovich and Longbrake, 2008; David et al., 2012a). However, they also secrete several factors that mediate cytotoxicity to neurons, glia, axons, and myelin (Popovich and Longbrake, 2008; David et al., 2012a). Therefore, the inflammatory response exerts both helpful and detrimental actions after SCI, so its final outcome on this pathology depends on the balance between mechanisms that regulate different aspects of the inflammatory response.

A self-limited inflammatory response is a prerequisite for a return to homeostasis (catabasis) and requires effective resolution of inflammation (Buckley et al., 2014; Serhan, 2014; Serhan et al., 2015). In contrast, insufficient or inadequate resolution leads to chronic inflammation that causes greater tissue damage, impaired tissue remodeling, and inappropriate tissue healing, such as pronounced deposition of extracellular matrix (Buckley et al., 2014; Serhan, 2014; Serhan et al., 2015). This is also the case after SCI, when inflammation fails to resolve properly, leading to disproportionate harmful bystander side effects (Hawthorne and Popovich, 2011; Prüss et al., 2011; David et al., 2012a). The damaging consequences of nonresolving inflammation are pronounced in the lesioned spinal cord due to the limited capacity of repair, such as axon regeneration and replacement of damaged neurons and myelin, leading to irreversible functional disabilities (Fawcett et al., 2012; Lu et al., 2014; Stenudd et al., 2015).

Resolution of inflammation is an active process regulated in part by a superfamily of lipid mediators derived from polyunsaturated fatty acids (PUFAs) (Schwab et al., 2007; David et al., 2012c; Serhan, 2014). This superfamily of specialized proresolving mediators (SPMs) include: lipoxins, resolvins [resolvin D $(\mathrm{RvD})$ and resolvin $\mathrm{E}(\mathrm{RvE})$ ], protectins, and maresins (Buckley et al., 2014; Serhan, 2014; Serhan et al., 2015). SPMs actively turn off the inflammatory response by acting on distinct G-proteincoupled receptors expressed on immune cells that activate dual anti-inflammatory and proresolution programs (Buckley et al., 2014; Serhan, 2014; Serhan et al., 2015). Among the antiinflammatory actions of SPMs include the induction in the

Received May 22, 2017; revised Sept. 27, 2017; accepted 0ct. 1, 2017.

Author contributions: I.F.Q., J.M.S., and R.L.-V. designed research; I.F.Q., E.S.-N., M.A.K., B.B., J.M.S., and R.L.-V. performed research; S.D. and J.M.S. contributed unpublished reagents/analytic tools; I.F.Q., K.G., A.B.S., M.A.K., B.B., and R.L.-V. analyzed data; S.D., J.M.S., and R.L.-V. wrote the paper.

This work was supported by the Spanish Ministry of Economy and Competitiveness (Grants SAF2010-17851 and SAF2013-48431-R to R.L-V.), the International Foundation for Research in Paraplegia (R.L-V.), and the Fondo de Investigación Sanitaria of Spain (TERCEL and CIBERNED; R.L-V.). J.M.S. received funding from the Wings for Life Spinal Cord Research Foundation, the Era-Net-NEURON Program of the European Union (SILENCE Grant \#01EW170A), NIDILRR (Grant \#90SI5020), and the W.E. Hunt \& C.M. Curtis Endowment. K.G. received funding from the National Institutes of Health (Grant EY026082).

I.F.Q., S.D., J.M.S., and R.L.-V. are inventors on a patent application filled by Universitat Autònoma de Barcelona ("Maresins for Use in the Treatment of CNS Injuries," PCT/IB2017/054398). The remaining authors declare no competing financial interests.

Correspondence should be addressed to either of the following: Ruben López Vales, Ph.D., Departament de Biologia Cellular, Fisiologia i Immunologia, Institut de Neurocie'ncies, Centro de Investigacio'n Biome'dica en Red sobre Enfermedades Neurodegenerativas, Universitat Auto'noma de Barcelona, 08193 Bellaterra, Catalonia, Spain, E-mail: Ruben.López@uab.cat; or Jan M. Schwab, M.D., Ph.D., The Neurological Institute, The Ohio State University, Wexner Medical Center, 395 W. 12th Ave, 7th Floor, Columbus, 0H 43210, E-mail: Jan.Schwab@osumc.edu.

DOI:10.1523/JNEUROSCI.1395-17.2017

Copyright $\odot 2017$ the authors $\quad 0270-6474 / 17 / 3711732-13 \$ 15.00 / 0$ expression of anti-inflammatory cytokines or inflammatory scavenging molecules such as IL-10, IL-1 decoy receptors, and IL-1 receptor antagonists (Buckley et al., 2014; Serhan, 2014). Conversely, SPMs activate specific mechanisms that trigger the resolution of inflammation, including the following: (1) downregulation of proinflammatory cytokines, (2) abrogation of intracellular pathways that lead to inflammation, (3) clearance of inflammatory cell detritus (such as apoptotic neutrophils) by macrophages, and (4) normalization of immune cells counts to basal levels also referred to as catabasis (Buckley et al., 2014; Serhan, 2014; Serhan et al., 2015). The importance of SPMs in the resolution of inflammation is evident in many chronic pathological conditions in which their production is insufficient, delayed, or even absent, and exogenous administration of SPMs reduces inflammation and mediates tissue protection (Schwab et al., 2007; Buckley et al., 2014). However, it is currently not known whether sustained inflammation in SCI is due to inadequate production of SPMs.

Here, we show that SPM biosynthesis is impaired after SCI and that systemic administration of Maresin 1 (MaR1) (Serhan et al., 2009), a docosahexaenoic acid (DHA)-derived SPM, is able to enhance resolution of inflammation, resulting in improved functional and histopathological outcomes. These results provide strong evidence about the beneficial effects of exogenous administration of MaR1 in a preclinical model of SCI and suggest that administration of SPMs could be a novel therapeutic approach to treat acute SCI in humans, for which there is currently no effective treatment.

\section{Materials and Methods}

SCI and MaR1 treatment. All surgical procedures were approved by the Universitat Autònoma de Barcelona Animal Care Committee and followed the guidelines of the European Commission on Animal Care. The methods for each procedure were performed in accordance with the approved guidelines. A total of 142 adult (8-10 weeks old) female C57BL/6 mice (Charles River Laboratories) were anesthetized with ketamine:xylazine $(90: 10 \mathrm{mg} / \mathrm{kg})$. After performing a laminectomy at the 11th thoracic vertebrae, the exposed spinal cord was contused using the Infinite Horizon Impactor device (Precision Scientific Instrumentation). Injuries were made using a force of 60 kilodynes and tissue displacement ranging between 500 and $700 \mu \mathrm{m}$, as described previously (Coll-Miró et al., 2016).

One hour after SCI, $1 \mu \mathrm{g}$ of MaR1 (7S,14S-dihydroxy-4Z, 8E, 10E, 12Z, 16Z, 19Z-DHA; Cayman Chemical) or vehicle was injected intravenously and then repeated daily thereafter until day 7 . The injected solution was always prepared from the MaR1 stock solution the day of the administration. This consisted of $1 \mu \mathrm{g}$ of MaR1 (10 $\mu \mathrm{l}$ of the stock solution) diluted in $100 \mu \mathrm{l}$ of saline at $37^{\circ} \mathrm{C}$. The solution was prepared in individual Eppendorf tubes (one per mouse) and injected within the first $15 \mathrm{~min}$ after its preparation. Vehicle solution consisted of $10 \mu \mathrm{l}$ of $100 \%$ alcohol in $100 \mu \mathrm{l}$ of saline at $37^{\circ} \mathrm{C}$. Although we delivered this solution intravenously, we now have data indicating that intraperitoneal administration is also effective. The MaR1 dosage was chosen accordingly to be above the levels of approved MaR1 bioactivity in experimental disease models (Serhan et al., 2015) and above the SPM dosages sufficient to exert bioactivity in the CNS with intact and closed blood-brain barrier (Marcheselli et al., 2003; Svensson et al., 2007).

Flow cytometry. To study the dynamics of immune cells in SCI, immune cells from the laminectomized and injured spinal cord were analyzed by flow cytometry at $1,3,7,14,21$, and $28 \mathrm{~d}$ postinjury (dpi) as described previously (Santos-Nogueira et al., 2015; Coll-Miró et al., 2016; Francos-Quijorna et al., 2016). Similarly, spinal cords from mice treated with MaR1 or saline were also harvested at day 1, 3, and 7 after lesion. Briefly, spinal cords were cut in small pieces and passed through a cell strainer of $70 \mu \mathrm{m}$ (BD Falcon) and the cell suspension was centrifuged twice at $300 \times g$ for $10 \mathrm{~min}$ at $4^{\circ} \mathrm{C}$. After cell counts, samples were 
divided and cells alone and isotype-matched control samples were generated to control for nonspecific binding of antibodies and for autofluorescence. The following antibodies from eBioscience were used at a 1:250 concentration: CD45-PerCP, CD11b-PE-Cy7, Ly6C-FITC, Ly6G-PE, Gr1-FITC, F4/80-APC or PE, CD3-FITC, CD4-APC, CD8-APC, CD19PE, CD206-FITC, and CD16/32-PE. After $30 \mathrm{~min}$ of incubation with combinations of antibodies at $4^{\circ} \mathrm{C}$, cells were fixed in $1 \%$ paraformaldehyde. For intracellular staining, cells were permeabilized with Permeabilization Wash Buffer (BioLegend), incubated with unconjugated rabbit antibodies against iNOS (1:200; Abcam), and goat antibodies against Arg1 (1:200; Santa Cruz Biotechnology) for $30 \mathrm{~min}$, followed by staining with Alexa Fluor 488- or Alexa Fluor 647-conjugated donkey secondary antibodies against rabbit or goat (1:500; Invitrogen) for $30 \mathrm{~min}$. Finally, samples were washed and fixed in $1 \%$ paraformaldehyde. To perform the analysis, cells were first gated for CD45 to ensure that only infiltrating leukocytes and resident microglia were selected. Then, a combination of markers were used to identify the following: for microglia, CD $45^{\text {low }}$, $\mathrm{CD} 11 \mathrm{~b}^{+}$, and $\mathrm{F} 4 / 80^{+}$; for macrophages, CD $45^{\text {high }}$, CD $11 \mathrm{~b}^{+}$, and F4/ $80^{+}$; for neutrophils, CD $45^{\text {high }}, \mathrm{CD} 11 \mathrm{~b}^{+}, \mathrm{F} 4 / 80^{-}$, and $\mathrm{Gr} 1^{\text {high }}$; for CD4 $\mathrm{T}$ cells, $\mathrm{CD} 45^{+}, \mathrm{CD}_{11 \mathrm{~b}}{ }^{-}, \mathrm{CD} 3^{+}$, and $\mathrm{CD} 4{ }^{+}$; for $\mathrm{CD} 8 \mathrm{~T}$ cells, $\mathrm{CD} 45^{+}$, $\mathrm{CD} 11 \mathrm{~b}, \mathrm{CD} 3^{+}$, and $\mathrm{CD} 8^{+}$; and for $\mathrm{B}$ cells, $\mathrm{CD} 45^{+}, \mathrm{CD}_{11 \mathrm{~b}}{ }^{-}, \mathrm{CD} 3^{-}$, and $\mathrm{CD} 19^{+}$. To study the phenotype of microglia and macrophages, these cells were further differentiated based on Ly6C, CD16/32, iNOS, CD206, and Arg1 expression (Coll-Miró et al., 2016; Francos-Quijorna et al., 2016). Analysis of inflammatory cell kinetics at the lesion site was conducted applying objective and quantifiable measures of resolution dynamics as described previously (Prüss et al., 2011). Cells were analyzed using FlowJo software on a FACSCanto flow cytometer (BD Biosciences).

Lipid mediator lipidomics. A $5 \mathrm{~mm}$ segment of uninjured and contused spinal cord centered on the lesion tissue was harvested at 1, 3, 7, and 14 dpi for LC-MS/MS. Briefly, for endogenous lipid autacoid analysis, frozen spinal cords were homogenized with a hand-held tissue grinder in $66 \%$ methanol $\left(4^{\circ} \mathrm{C}\right)$. Homogenized tissue samples were combined with 2 volumes of methanol $\left(4^{\circ} \mathrm{C}\right)$. The methanol contained deuterated internal standards, PGE2-d4, lipoxin $\mathrm{A}_{4}\left(\mathrm{LXA}_{4}\right)$-d5, leukotriene B4 ( $\mathrm{LTB}_{4}-$ d4), 15(S)-hydroxyeicosatetraenoic acid [15(S)-HETE-d8], arachidonic acid (AA)-d8, and DHA-d54 at $400 \mathrm{pg} /$ each to calculate recovery of different classes of oxygenated fatty acids and PUFAs. Lipid autacoids were extracted by solid phase using Accubond ODS-C18 cartridges (Agilent Technologies). Eicosanoids, docosanoids, and PUFAs were identified and quantified by LC/MS/MS-based lipidomics (Hassan and Gronert, 2009; Prüss et al., 2013). In brief, extracted samples were analyzed by a triple quadruple linear ion trap LC/MS/MS system (MDS SCIEX 3200 QTRAP) equipped with a LUNA C18-2 mini-bore column using a mobile phase (methanol:water:acetate, 65:35:0.02, v:v:v) with a $0.50 \mathrm{ml} /$ flow rate. MS/MS analyses were performed in negative ion mode and hydroxy fatty acids were quantified by multiple reaction monitoring using established transitions. Calibration curves (1-1000 pg) and specific LC retention times for each compound were established with synthetic standards (Cayman Chemical).

Cytokine protein expression. Mice treated with saline or MaR1 were perfused with sterile saline and a $5 \mathrm{~mm}$ length of spinal cord centered on the lesion was collected at 12 and $24 \mathrm{~h}$ after contusion injury and snap frozen. Spinal cords were homogenized and protein concentration was determined using the DC Protein Assay (Bio-Rad). Samples were concentrated to $4 \mu \mathrm{g} / \mu \mathrm{l}$ using MicroCon centrifugation filters (Millipore) to ensure equal amounts of protein. Low concentrations of cytokines in the sample result in binding to the filters, whereas high concentrations of protein sustain fewer losses. The protein levels of 32 cytokines and chemokines were then analyzed using the Milliplex MAP Mouse Cytokine/Chemokine magnetic bead panel (Millipore) on a Luminex (Millipore) as per the manufacturer's protocol (Francos-Quijorna et al., 2016).

Western blotting. Samples used for the Luminex assay were also used for Western blotting. Protein samples $(30 \mu \mathrm{g})$ were separated by electrophoresis on a $10-15 \%$ polyacrylamide gel and transferred onto PVDF membranes (Millipore). The membranes were incubated overnight at $4^{\circ} \mathrm{C}$ with rabbit antibodies against phospho NF- $\kappa$ B p65 (1:1000; Cell Signaling Technology), against the phosphorylated form of STAT1 (1: 500; Cell Signaling Technology), STAT3 (1:500; Cell Signaling Technol- ogy), STAT5 (1:500; Cell Signaling Technology) and STAT6 (1:500; Cell Signaling Technology), JNK (1:500; Santa Cruz Biotechnology), ERK1/2 (1:1000; Cell Signaling Technology), p38 (1:1000; Cell Signaling Technology), and AKT (1:1000; Cell Signaling Technology). Bands were detected using chemiluminescence (Immobilon Western Chemiluminescence HRP reagent; Millipore) and data were quantified by densitometry using Workflow version 3 software in a Chemidoc apparatus (Millipore). $\beta$-Actin (1:10.000; Sigma-Aldrich) was used to ensure equal loading of samples.

Functional assessment. Locomotor recovery was evaluated at 1, 3, 5, 7, $10,14,21$, and $28 \mathrm{~d}$ postinjury (dpi) in an open-field test using the nine-point Basso Mouse Scale (BMS) (Basso et al., 2006), which was developed specifically for locomotor testing after contusion injuries in mice. The BMS analysis of hindlimb movements and coordination was performed by two independent assessors blinded to the treatment groups (MaR1 vs saline) and the consensus score taken. In addition, at the end of the follow-up (28 dpi), a computerized assessment of locomotion was also performed using the DigiGait Imaging System (Mouse Specifics). This system consists of a motorized transparent treadmill belt and a high-speed digital video camera that captures images of the paws from the underside of the walking animals. DigiGait software generates "digital pawprints" and dynamic gait signals, representing the temporal record of paw placement relative to the treadmill belt. This locomotor test allows for an easy and objective analysis of both static and dynamic locomotor parameters. Moreover, the highest locomotion speed that each mouse was able to locomote for at least $5 \mathrm{~s}$ was also recorded on the DigiGait treadmill belt. Functional tests were done blinded to the experimental groups.

Histology. At 28 dpi, mice were perfused with $4 \%$ paraformaldehyde in $0.1 \mathrm{M}$-phosphate buffer (PB). A $5 \mathrm{~mm}$ length of spinal cord containing the lesion site was removed, cryoprotected with $30 \%$ sucrose in $0.1 \mathrm{M} \mathrm{PB}$ at $4^{\circ} \mathrm{C}$, and 10 series of $10-\mu \mathrm{m}$-thick sections were picked up on glass slides. Adjacent sections on the same slide were therefore $100 \mu \mathrm{m}$ apart. For quantification of myelin area content in the spinal cord analyses, sections were stained with Luxol Fast Blue (LFB; Sigma). For neuronal and axonal assessment, sections were incubated overnight at $4^{\circ} \mathrm{C}$ with biotinylated antibodies against NeuN (1:200; Millipore) and NF (1:1000; Millipore), respectively. Double immunostaining for NF and myelinbinding protein (MBP, 1:100; Abcam) was done to assess the sparing of myelinated axons. Sections were incubated for $1 \mathrm{~h}$ at room temperature with the streptavidin/Alexa Fluor 594-conjugated or donkey anti-rabbit Alexa Fluor 594-conjugated antibodies (1:500; Invitrogen) and then coverslipped in Mowiol containing DAPI to label nuclei.

The epicenter of the injection or contusion injury impact was determined for each mouse spinal cord by localizing the tissue section with the greatest damage using an LFB-stained section. Myelin content after SCI was calculated by delineating the area of LFB-stained tissue. Neuronal survival was assessed by counting the number of $\mathrm{NeuN}^{+}$cells in the ventral horns at the injury epicenter and at rostral and caudal areas. Axonal sparing was calculated by counting the number of axons in the dorsal column at the injury epicenter, the region of most pronounced damage. The same sections were used to examine axonal demyelination in the dorsal column through counting the fibers double stained for NF and MBP at the lesion epicenter. All quantifications were performed blinded to the experimental groups with the help of the ImageJ image analysis software.

Statistical analyses. Data are shown as mean \pm SEM. The Kolmogoro$\mathrm{v}-$ Smirnov test was used to test normality. Dependent on data being normally or non-normally distributed, we chose parametric or nonparametric tests. Dynamics of immune cell recruitment and lipidomic profile after SCI were analyzed with one-way ANOVA with post hoc Bonferroni's test. Functional follow-up for BMS score and subscore, as well as histological analysis of myelin and neuronal sparing, were analyzed using two-way repeated-measures ANOVA with post hoc Bonferroni's post hoc test for multiple comparisons. Two-tailed Student's or the nonparametric Mann-Whitney's test was used for single comparisons between two groups and one-way ANOVA followed by Dunnett's multiple post hoc test for comparisons of more than two groups. Maximal speed on a 
A
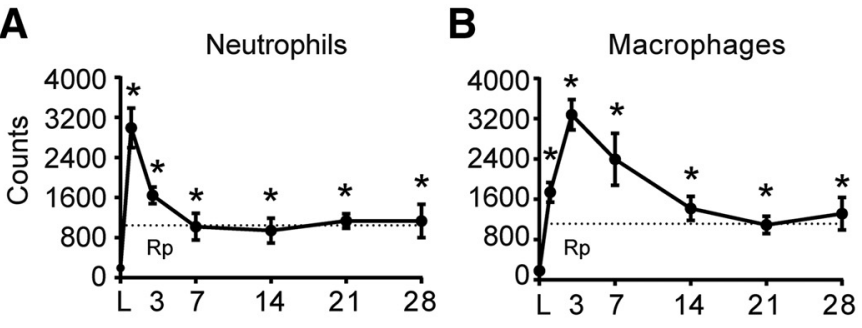

D

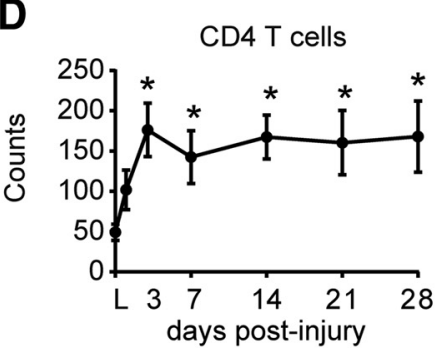

E

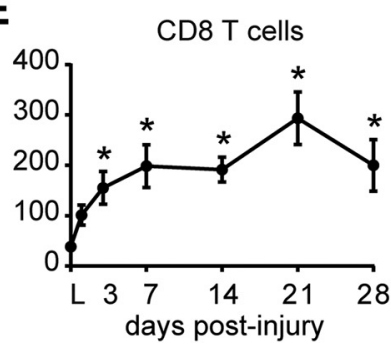

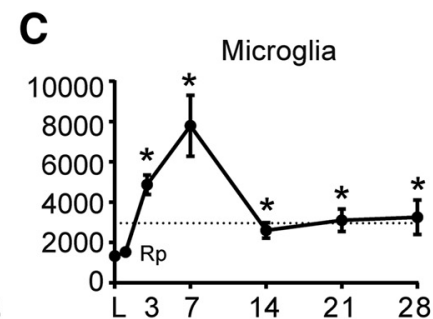

$\mathbf{F}$

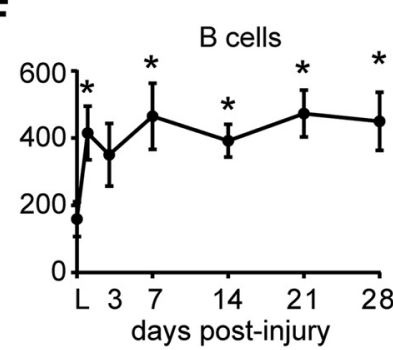

Figure 1. Temporal dynamics of changes in leukocyte numbers at the lesion site after SCl in mice. $\boldsymbol{A}-\boldsymbol{F}$, Graphs showing neutrophil (A), macrophage (B), microglial (C), CD4 T-cell (D), CD8 T-cell $(\boldsymbol{E})$, and B-cell $(\boldsymbol{F})$ kinetics in the contused spinal cord for the first 4 weeks. Note that the counts for the different immune cell populations remained elevated throughout this period. Dashed lines indicate the resolution plateau (Rp). ${ }^{*} p<0.05$ versus laminectomy (L). One-way ANOVA with Tukey's post hoc correction ( $n=8$ per point). Error bars indicate SEM.

treadmill was analyzed using the Mantel-Cox test. Differences were considered significant at $p<0.05$.

\section{Results}

\section{Inflammatory cell clearance is impaired after SCI}

We first evaluated, using flow cytometry, the dynamics of the main inflammatory cell types in the contused spinal cord of C57BL/ 6 mouse and assessed different parameters to characterize the inflammatory resolution and to determine the persistence of the different immune cell subpopulations at the lesion site after spinal cord contusion in mice. We detected that the accumulation of neutrophils, macrophages, and microglia cell reached maximal cell numbers in the contused spinal cord at 1,3 , and 7 dpi, respectively (Fig. $1 A-C$ ). Subsequently, their numbers dropped progressively up to 7-14 dpi, remaining at high and steady levels up to $28 \mathrm{dpi}$ (Fig. $1 A-C)$. The resolution index $\left(R_{\mathrm{i}}\right.$, the window between the time point of maximum cell numbers to a reduction by $50 \%$ ) of neutrophils and macrophages was 2.5 and $9.5 \mathrm{~d}$, respectively, reflecting the slower clearance of macrophages in SCI compared with neutrophils. Microglia $R_{\mathrm{i}}$ could not be calculated because of the rapid decline in their cell counts from 7 to $14 \mathrm{dpi}$; however, it was $<7$. We then quantified the resolution plateau $\left(R_{\mathrm{p}}\right.$, the percentage of persistent cellular component relative to maximum cell numbers) to provide quantitative measurements of the inflammatory resolution after SCI (Prüss et al., 2011). $R_{\mathrm{p}}$ revealed that the clearance of all three myeloid cell subsets after SCI was incomplete, with $\sim 35 \%$ remaining neutrophils, macrophages, and microglial cells at $28 \mathrm{dpi}$ (Fig. 1A-C).

We also studied the recruitment of lymphocytes in the contused spinal cord. We observed infiltration of B cells and T cells, both $\mathrm{CD} 4{ }^{+}$and $\mathrm{CD} 8{ }^{+}$lymphocytes, during the first few days after contusion injury, but at much lower numbers compared with monocytes (Fig. $1 D-F$ ). $R_{\mathrm{p}}$ of the different lymphocyte subsets was $>50 \%$ at day 28 , indicating the persistent presence of lymphocytes in SCI exposed to CNS antigens throughout. These results provide clear evidence that immune cells are not eliminated efficiently from the contused spinal cord and highlight that the resolution capacity of the injured spinal cord is impaired after SCI.
Defective lipid mediator class switch as a classical hallmark of impaired resolution in acute SCI lesions

We investigated whether the impaired clearance of inflammatory cells is mirrored by failed induction of synthesis of SPMs, which have been identified as being crucial for efficient resolution (Serhan, 2014). Lipidomic analysis of spinal cord revealed delayed synthesis of SPM after contusion injury. The levels of 12-HETE and 15-HETE, which are pathway markers of the synthesis of the AA-derived SPMs known as $\mathrm{LXA}_{4}$, did not increase until $14 \mathrm{dpi}$ (Fig. 2; one-way ANOVA, Bonferroni's post hoc test, $F_{(4,15)}=$ 5214 for 12-HETE; $F_{(4,15)}=4074$ for 15-HETE $\left.p<0.05\right)$ Levels of 5-HETE, however, did not change after injury (Fig. 2). Similarly, the synthesis of SPMs derived from DHA was also delayed in SCI because the levels of 17-HDHA, a pathway marker for the formation of $\mathrm{RvD}$ and protectin $\mathrm{D} 1$ (PD1), and 14-HDHA, the pathway marker for the biosynthesis of MaR1, were not induced until day 14 (one-way ANOVA, Bonferroni's post hoc test, $F_{(4,15)}=$ 2475 for 17-HDHA; $F_{(4,15)}=4174$ for 14-HDHA; $\left.p<0.05\right)$. Moreover, SPM derived from eicosopentaenoic acid (EPA) were also impaired after SCI because 18-HEPE, the pathway marker for the formation of the RvE series, was undetected in the injured spinal cord for the time period analyzed $(14 \mathrm{~d})$. Therefore, the CNS lesion milieu is characterized by a defective and delayed induction of SPMs involving those derived from the AA $(\omega-6)$, DHA, and EPA ( $\omega-3)$ pathways, which are required for orchestrating efficient resolution of inflammation. This inability to generate a resolution conducive milieu is contrasted by a full-blown early PGE2 response as a hallmark of proinflammatory activity (one-way ANOVA, Bonferroni's post hoc test, $F_{(4,15)}=10,14 ; p<$ 0.05 ; Fig. 2). These data indicate that the class switch from proinflammatory to proresolution lipid mediators derived from AA, DHA, and EPA does not occur properly in the injured spinal cord.

\section{MaR1 regulates resolution of inflammation in the injured spinal cord}

To assess whether the deficit in the resolution of inflammation after SCI is linked to impaired synthesis of SPM, we investigated 

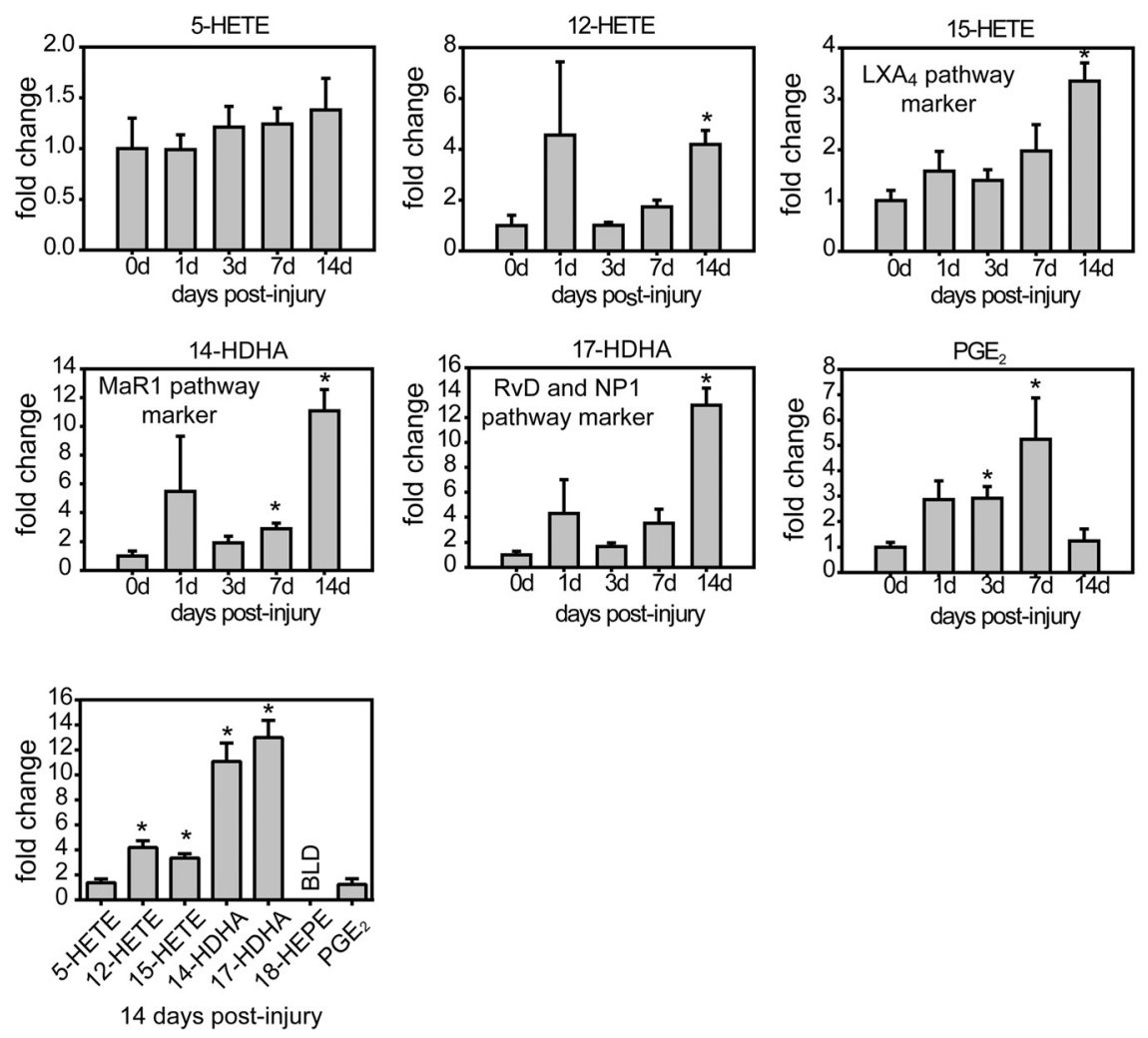

Figure 2. Impaired and delayed synthesis of proresolving lipid mediators after SCl. Resolution metabolome profiles after SCI were analyzed using LC-MS/MS of whole spinal cord lysates. Proinflammatory eicosanoid profiles indicated by $\mathrm{PGE}_{2}$ levels follow closely the formation of inflammation, with an early increase at day 1 , increasing to peak at day 7 , followed by a drop until day 14 . This time point marks a switch in the lipid mediator biosynthesis profile demarcated by starting increase of the proresolution pathways. Both MaR1 and RvD synthesis commences not before 2 weeks after $\mathrm{SCl}$ as indicated by a 10- to 13 -fold increase of the pathway markers such as 14-HDHA (MaR1) or 17-HDHA (RvD and NP1). This is further matched by the weak and late induction of 5-HETE, 12-HETE, and 15-HETE, which are indicative of the biosynthesis of the SPM LA 4 . Note that at 14 dpi, proresolving pathways are increased significantly in the injured spinal cord, with the exception of RvE, the levels of which were below the limit of detection (BLD). Conversely, the levels of the proinflammatory eicosanoid PGE 2 were not increased at this time point. ${ }^{*} p<0.05$ versus uninjured spinal cords ( 0 d). One-way ANOVA with Bonferroni's post hoc correction ( $n=4$ per point). Error bars indicate SEM.
These results provide clear evidence that systemic delivery of MaR1 enhances the elimination of peripheral myeloid cells (neutrophils and macrophages) from the injured spinal cord, suggesting an important role for MaR1 in promoting resolution of inflammation after SCI.

\section{MaR1 silences cytokine expression in SCI}

In an attempt to assess the mechanisms underlying the resolving actions of MaR1 in SCI, we assessed changes in expression of cytokines at the protein level in the contused spinal cord using a Luminex assay. These experiments revealed that MaR1 treatment reduced the levels of CXCL1, CXCL2, CCL3, CCL4, IL-6, and CSF3 significantly (one-way ANOVA, Dunnet's post hoc test, $F_{(3,14)}=7.78, p<0.05$; Fig. $5 A$; Table 1). In addition, the expression of IL-3, IL-13, and CXCL5, which were found at low levels in contused spinal cords of mice treated with vehicle, were undetectable in those treated with MaR1 (Table 1). IL-4 protein levels were undetected in the injured spinal cord of both groups. Note that MaR1 did not reduce the protein levels of the anti-inflammatory cytokine IL-10 after SCI (Fig. 5A), suggesting that MaR1 attenuates proinflammatory cytokines preferentially.

Because cytokines are regulated by multiple signal transduction pathways, we then investigated which of the main inflammatory signaling mechanisms were attenuated by MaR1 after SCI. Western blot analysis of spinal cord tissue taken whether systemic administration of the DHA-derived SPM called MaR1 enhanced immune cell clearance from the contused spinal cord. We first assessed the effects of MaR1 on neutrophil dynamics (two-way ANOVA, Bonferroni's post hoc test; effect of day $F_{(3,40)}=63.68, p<0.0001$; effect of treatment $F_{(1,40)}=3.424, p=$ $0.0317)$. Daily intravenous administration of MaR1 for $7 \mathrm{~d}$ starting $1 \mathrm{~h}$ after SCI did not impede the infiltration of neutrophils into the contused spinal cord because their counts at $1 \mathrm{dpi}$, when neutrophil accumulation peaks after SCI, were unaltered by MaR1 treatment (Fig. 3). However, MaR1 accelerated the clearance of neutrophils from the contused spinal cord based on several resolutions parameters $\left(R \mathrm{i}\right.$ and $\left.T_{50}\right)$, and reduced $\sim 50 \%$ the neutrophil counts in the injured spinal cord at day 7 (Fig. 3). We next studied whether MaR1 interfered with the recruitment of macrophages after SCI (two-way ANOVA, Bonferroni's post hoc test; effect of day $F_{(3,40)}=57.39, p<0.0001$; effect of treatment $\left.F_{(1,40)}=3.932, p=00543\right)$. The entrance of bloodborne macrophages into the contused spinal cord was not different at day 1 after MaR1 treatment (Fig. $4 A-C$ ), but tended to be reduced at 3 dpi, although not significantly. However, macrophage accumulation in the lesioned spinal cord was reduced significantly after MaR1 treatment at 7 dpi (Fig. $4 A-C$ ). MaR1 treatment did not attenuate microglial numbers in the contused spinal cord during the first week after contusion injury, although it tended to be reduced at 7 dpi upon administration of this SPM (Fig. $4 D$ ).
$24 \mathrm{~h}$ after SCI revealed that levels of pP65 and pAkt were upregulated after contusion injury, but these levels were not affected by MaR1 treatment (one-way ANOVA, Dunnet's post hoc test, $F_{(2,7)}=2.44, p=0.562$; Fig. $\left.5 B, C\right)$. In contrast, the STAT and MAPK pathways, two of the main inflammatory signaling mechanisms, showed differences after SCI. Specifically, STAT1, STAT3, and STAT5, as well as p38 and ERK1/2, were increased significantly at $24 \mathrm{~h}$ after injury in saline-treated mice and all of them were attenuated upon MaR1 treatment (one-way ANOVA, Dunnet's post hoc test, $F_{(2,7)}, p<0.05$; Fig. $\left.5 B, C\right)$. STAT6 and $\mathrm{JKN}$, which were not activated significantly after SCI, remained unaltered after MaR1 administration. These data provide clear evidence that MaR1 silences cytokine expression and turns off the activation of some members of the STAT and MAPK proinflammatory signaling pathways, but does not limit NF- $\kappa \mathrm{B}$ and PI3K/Akt signaling after SCI.

\section{Actions of MaR1 on microglia and macrophage after SCI}

Macrophages are a heterogeneous population of cells that exert divergent effects on damaged tissue depending on their phenotype. Ly6 $\mathrm{C}^{\text {high }}$ macrophages are proinflammatory and exhibit phagocytic, proteolytic functions and mediate cytotoxicity. In contrast, Ly6C ${ }^{\text {low }}$ (also known as LyC6 ${ }^{\text {neg }}$ ) macrophages are antiinflammatory and promote wound healing and repair (Arnold et al., 2007; Nahrendorf et al., 2007). Because cytokines play a key 
A

Saline
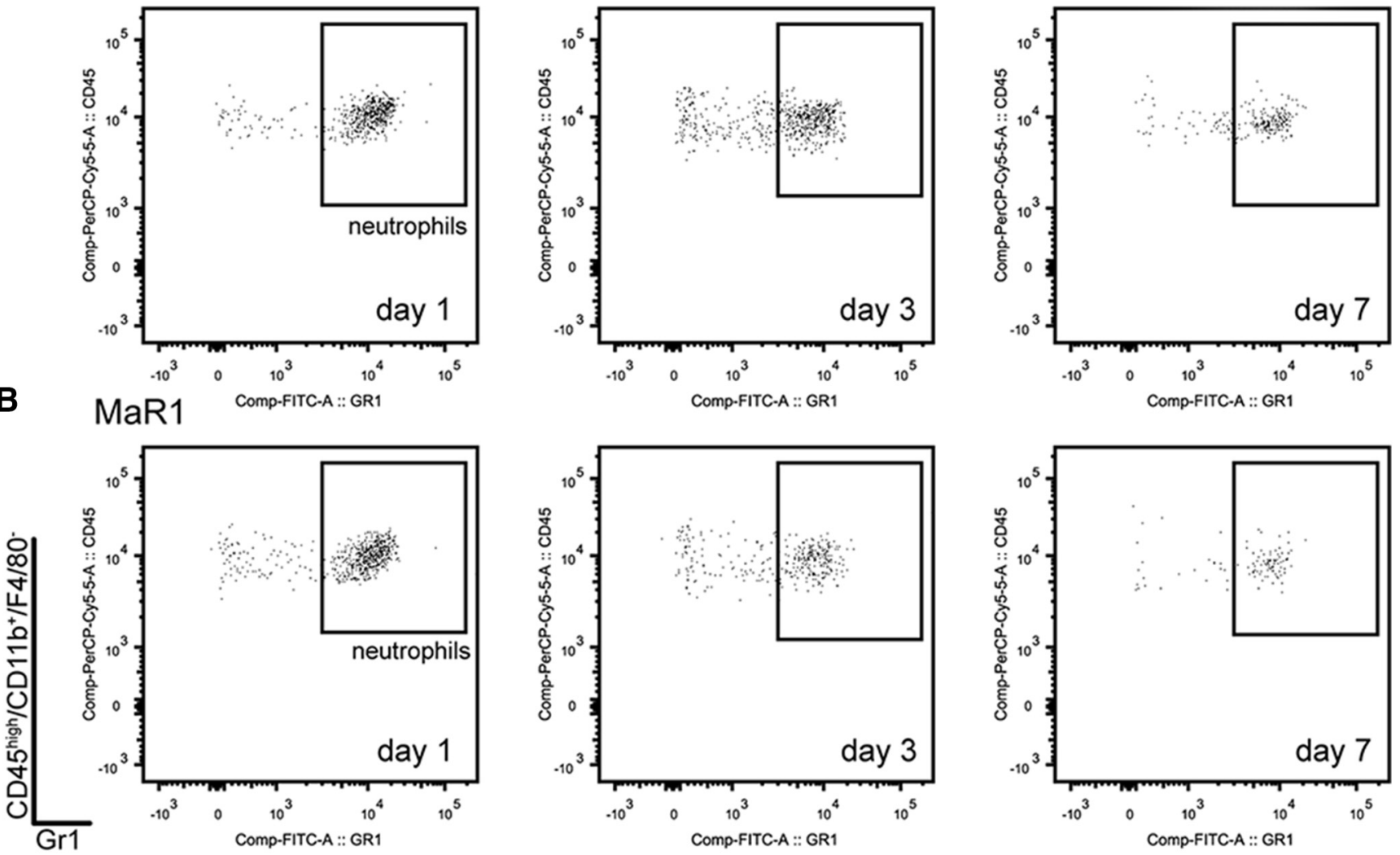

C

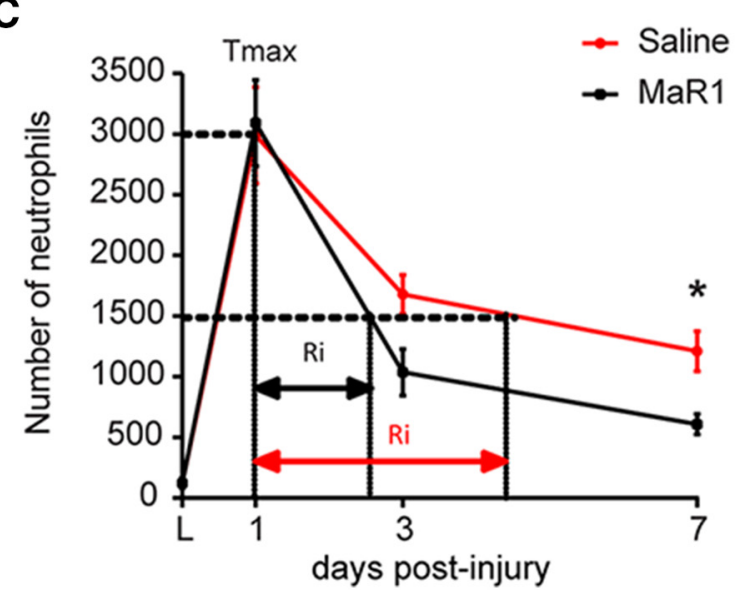

Time (d)

\begin{tabular}{|c|c|c|c|}
\hline $\begin{array}{c}\text { Resolution } \\
\text { Indices }\end{array}$ & $\begin{array}{c}\mathrm{SCl} \\
\text { Saline } \\
\end{array}$ & $\begin{array}{c}\mathrm{SCl} \\
\mathrm{MaR1} \\
\end{array}$ & $\%$ reduction \\
\hline$\Psi \max \left(10^{3}\right)$ & $2.99 \pm 0.40$ & $3.09 \pm 0.35$ & - \\
\hline$\Psi 3 \mathrm{dpo}\left(10^{3}\right)$ & $1.68 \pm 0.16$ & $1.04 \pm 0.19$ & 38.25 \\
\hline Y7dpo $\left(10^{3}\right)$ & $1.21 \pm 0.16$ & $0.61 \pm 0.08$ & $49.72 *$ \\
\hline$T \max (h)$ & 24 & 24 & - \\
\hline T50 (h) & $\sim 106$ & $\sim 61$ & $\sim 42.5$ \\
\hline $\mathrm{Ri} \quad(h)$ & $\sim 82$ & $\sim 37$ & $\sim 54.9$ \\
\hline
\end{tabular}

Figure 3. MaR1 propagates the resolution of neutrophil inflammation. $A, B$, Representative density plots of $F A C S$ analysis showing neutrophils at 1,3 , and $7 \mathrm{~d}$ after the injury in the spinal cord of saline- $(\boldsymbol{A})$ and MaR1 $(\boldsymbol{B})$-treated mice. $\boldsymbol{C}$, Graph showing neutrophil recruitment and resolution indices. Note that MaR1 treatment (red line) does not interfere with the proinflammatory cell infiltration, but induces a more rapid decline of neutrophils. Inset shows some inflammatory kinetics measurement, which include: $\psi \max =\operatorname{maximal}$ cell counts; $\psi 3$ and $\psi 7=$ cell counts at 3 and $7 \mathrm{dpi} ; T_{\text {max }}$ time after $S \mathrm{Cl}$ until reaching max cell numbers, $T_{50}$, time after $\mathrm{SCl}$ until reduction of cell numbers by $50 \%$; and $R_{\mathrm{i}}{ }^{*} p<0.05$ versus saline. Two-way ANOVA with Bonferroni's post hoc test was used to analyze significant differences in the dynamics of neutrophil counts after $\mathrm{SCl} ; t$ test was used to assess the different inflammatory kinetic indices ( $n=6$ per time point and group). Error bars indicate SEM.

role in regulating macrophage phenotype (David and Kroner, 2011; Kroner et al., 2014), we investigated whether MaR1 modulated the proportion of Ly6 $\mathrm{C}^{\text {high }}$ and Ly $6 \mathrm{C}^{\text {low }}$ macrophages at $7 \mathrm{~d}$ after SCI, the time point when MaR1 treatment reduced the number of these cells. We found that MaR1 had a significant impact on macrophage phenotype based on Ly6C expression because this SPM reduced $(\sim 50 \%)$ the amount of proinflammatory macrophages (Ly6C ${ }^{\text {high }}$ ) markedly, but not the anti-inflammatory macrophages (Ly6C ${ }^{\text {low }}$ ) (Fig. 6A,B). Indeed, the ratio of $\mathrm{LyC6}^{\text {low }}$ LyC6 ${ }^{\text {high }}$ in saline-treated SCI mice was $1.57 \pm 0.39$. In contrast, this ratio was increased to $3.73 \pm 0.26$ by MaR 1 ( $t$ test, $t=4.201 ; \mathrm{df}=10$; $p=0.0036)$, highlighting that there were $\sim 4$-fold greater antiinflammatory than proinflammatory macrophages in the spinal cord of mice treated with MaR1 (Fig. $6 A, B$ ).

This SPM also reduced expression of the proinflammatory, cytotoxic enzyme iNOS significantly (Fig. 6C,E) in macrophages 


\section{A Saline}
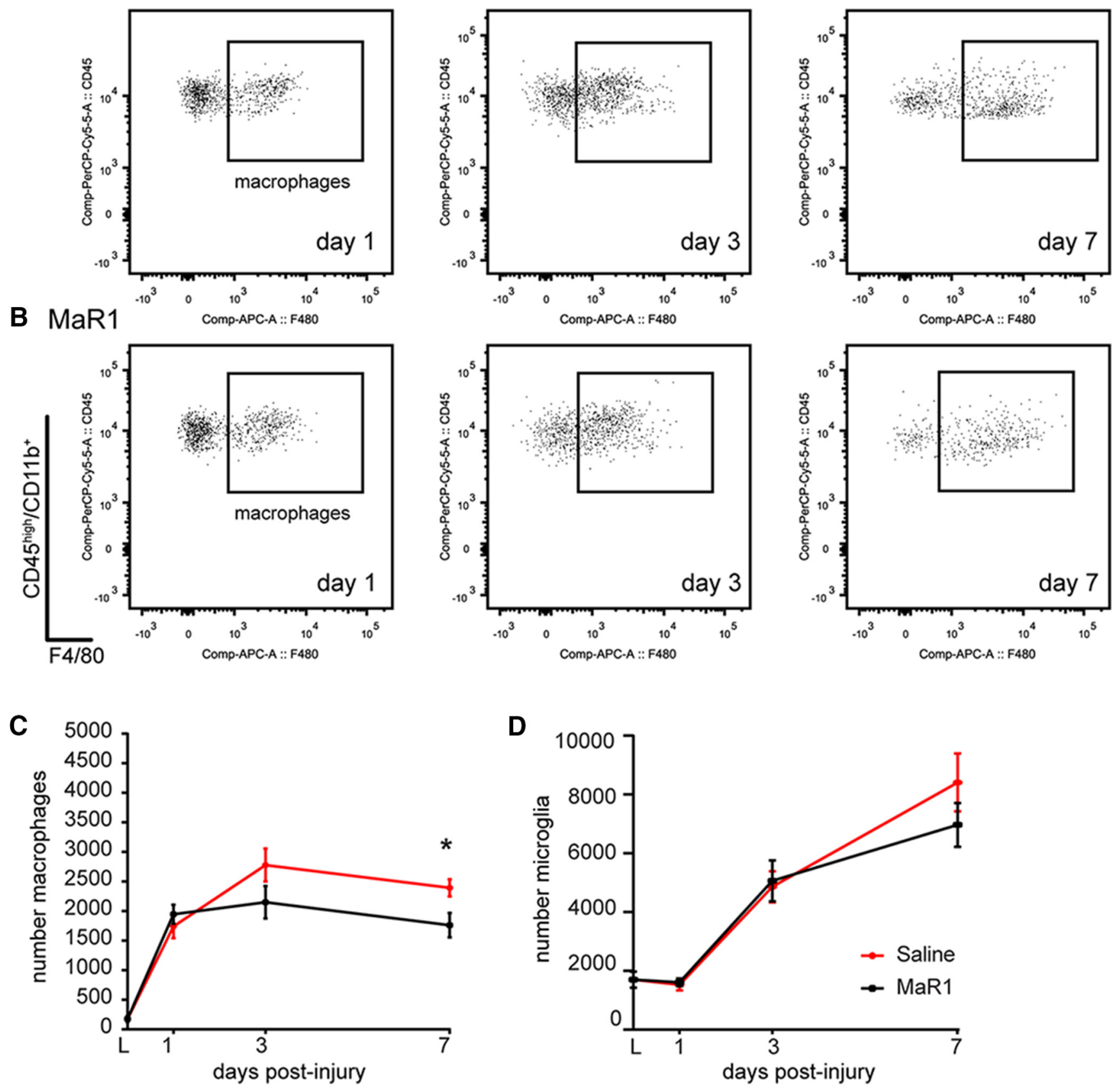

Figure 4. MaR1 propagates late macrophage clearance from the lesion site. $A, B$, Representative FACS analysis dot plots showing the dynamics of macrophages accumulation in the spinal cord at 1,3, and 7 dpi in saline- $(\boldsymbol{A})$ and MaR1 $(\boldsymbol{B})$-treated mice. $\boldsymbol{C}, \boldsymbol{D}$, Graphs showing quantification of macrophage and microglial cells from FACS analysis. Note the reduced numbers of macrophages at day 7 after MaR1 treatment, demarcating the enhanced resolution plateau triggered by this SPM. However, microglial counts were not modulated by MaR1 for the first week after injury. ${ }^{*} p<$ 0.05 versus saline. Two-way ANOVA with Bonferroni's post hoc test was used to analyze significant differences ( $n=6$ per time point and group). Data are shown as mean \pm SEM.

( $t$ test, $t=4.779 ; \mathrm{df}=10 ; p=0.0007)$. Moreover, MaR1 induced a $\sim 2$-fold increase of Arg-1 expression in macrophages, which was barely detectable in vehicle controls, although it did not reach statistical significance ( $t$ test, $t=1.288$; $\mathrm{df}=10 ; p=0.2266$; Fig. $6 C, E)$. Together, these data indicate that MaR1 converts the phenotype of macrophages in the injured spinal cord toward a more prorepair and anti-inflammatory state.

In contrast to macrophages, most microglial cells were Ly6C ${ }^{\text {low }}$ in SCI $(\sim 85 \%)$ and MaR1 did not reduce the percentage of Ly6C $\mathrm{C}^{\text {high }}$ microglia $(9.6 \pm 0.8 \%$ and $10.6 \pm 1.1 \%$ in saline- and MaR1-treated mice, respectively). MaR1 treatment tended to re- duce the expression of iNOS (Fig. 6D,F), although not to a statistically significant level. These results, therefore, suggest that the immunomodulatory effects of MaR1 after SCI are mostly related to macrophages but not microglia, at least up to $7 \mathrm{dpi}$.

Because phagocytosis of neutrophils by macrophages is a crucial step for the resolution of inflammation (Schwab et al., 2007; Serhan, 2014; Serhan et al., 2015), we determined whether MaR1 increased the ability of macrophages to phagocytose neutrophils (efferocytosis). We found that the amount of the selective neutrophil marker Ly6G inside the macrophages $\left(\mathrm{CD} 45^{\text {high }}, \mathrm{CD} 11 \mathrm{~b}{ }^{+}, \mathrm{F} 4 /\right.$ $80^{+}$) was increased $\sim 2$-fold in the spinal cords of mice treated 
A

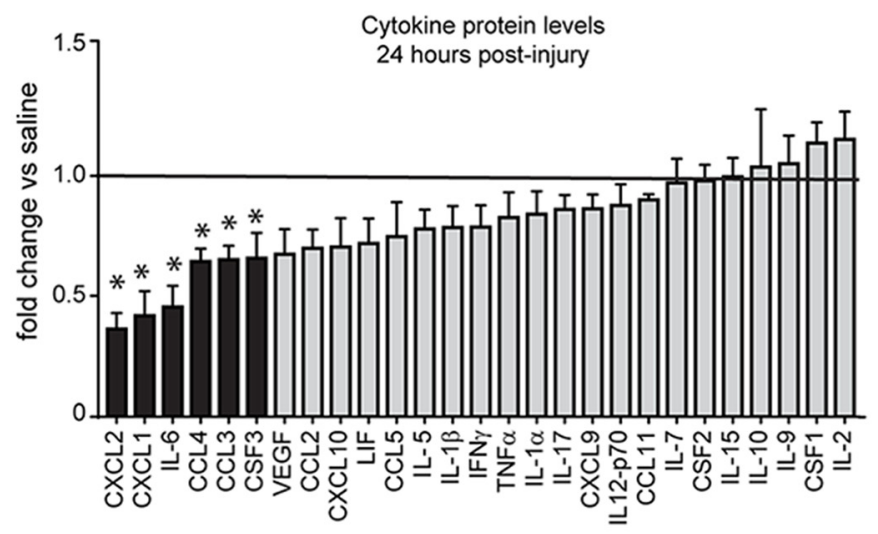

B

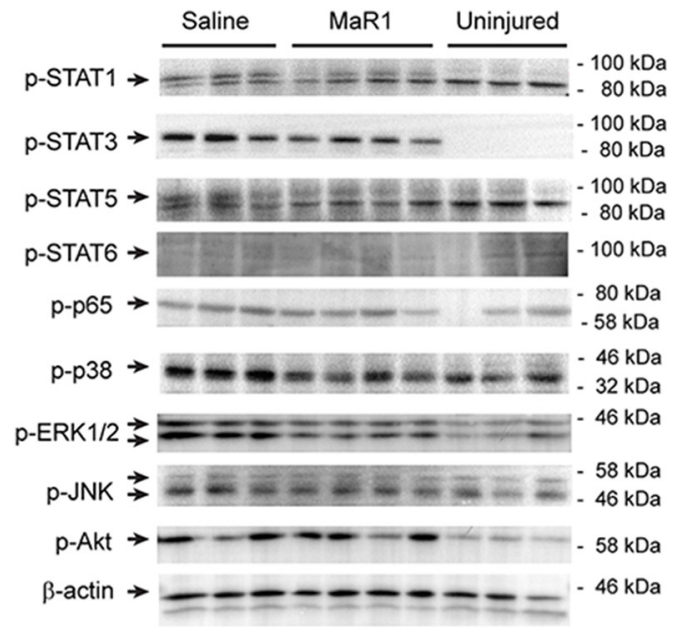

C

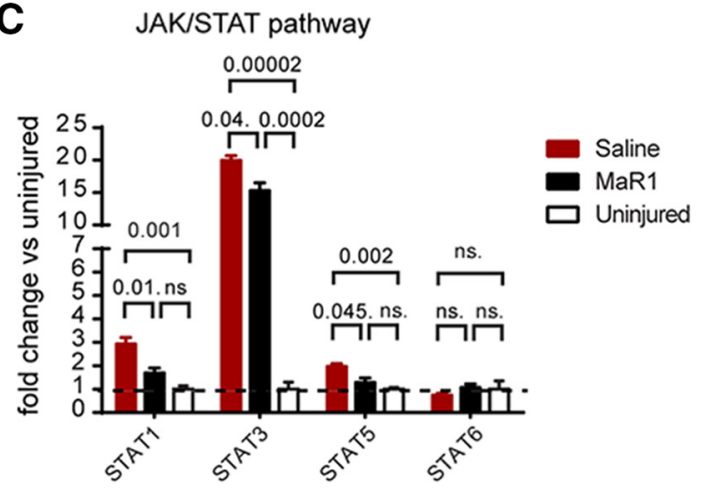

NF-kB pathway MAPK pathway

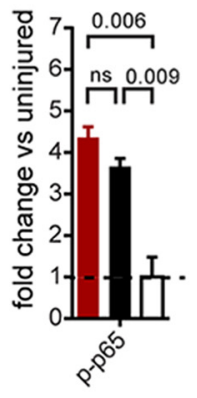

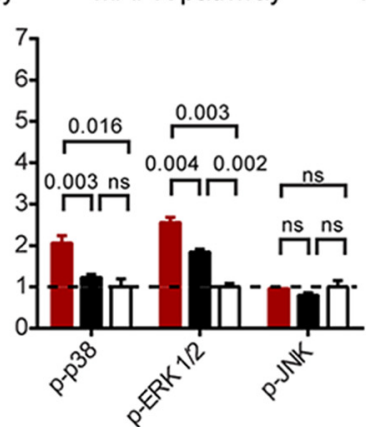

PI3K/Akt pathway

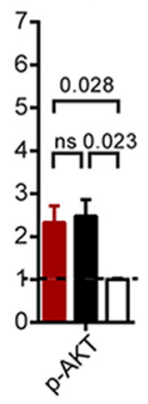

Figure 5. Acute mechanistic signaling underlying systemic MaR1 treatment at the lesion site. $A$, Cytokine protein level profile $24 \mathrm{~h}$ after MaR1 treatment is characterized by a reduced expression of chemokines (CXC12, CXCL1, CCL3, CCL4, and CSF3) and the proinflammatory cytokine IL-6 (black bars) as indicated by Luminex analysis. B, C, Western blot analysis showing different inflammatory intracellular pathways in contused spinal cord at $24 \mathrm{~h}$ after injury. Note that MaR1 treatment attenuated the activation of STAT-1, STAT-3, STAT-5, p38, and ERK1/2 signaling at the lesion site, but does not limit NF- $\kappa$ B and PI3K/Akt activation after $S C I .{ }^{*} p<0.05$ versus saline. One-way ANOVA with Dunnet's post hoc test was used to analyze significant differences $(n=3$ in uninjured and contused saline-treated injured mice; $n=4$ in contused MaR1-treated mice). Data are shown as mean \pm SEM.

Table 1. Protein levels of cytokines silenced significantly by MaR1 in SCI

\begin{tabular}{llcl}
\hline & Naive & Saline & MaR1 \\
\hline IL-3 & ND & $0.56 \pm 0.03$ & ND \\
IL-6 & $0.94 \pm 0.03$ & $671 \pm 133$ & $291 \pm 56.7^{*}$ \\
IL-13 & ND & $4.19 \pm 1.38$ & ND \\
CSF3 & ND & $1179 \pm 182$ & $741 \pm 118^{*}$ \\
CXCL1 & $2.81 \pm 0.23$ & $219 \pm 67.6$ & $87.5 \pm 21.3^{*}$ \\
CXCL2 & $4.59 \pm 0.30$ & $119 \pm 17.9$ & $41.4 \pm 7.50^{*}$ \\
CXCL5 & ND & $4.80 \pm 0.86$ & ND \\
CCL3 & ND & $21.5 \pm 2.52$ & $13.3 \pm 1.18^{*}$ \\
CCL4 & ND & $28.9 \pm 3.47$ & $17.7 \pm 1.46^{*}$ \\
\hline
\end{tabular}

${ }^{*} p<0.05$, MaR1 versus saline.

ND, Not detectable (i.e., below the limits of detection). with MaR1 at 7 dpi (Mann-Whitney test, $p=0.0286)$, indicating that this SPM enhanced neutrophil phagocytosis in SCI (Fig. $7 A, B)$. Therefore, exogenous administration of MaR1 drives macrophage activation toward a more restorative phenotype after SCI and enhances efferocytosis.

Administration of MaR1 reduces tissue damage and improves locomotor recovery after SCI

Finally, we investigated whether MaR1 improves functional and histological outcomes after SCI. Mice treated with MaR1 demonstrated significant improvement in locomotor recovery, resulting in elevated BMS scores (two-way ANOVA, Bonferroni's post hoc test; effect of day $F_{(8,36)}=129.5, p<0,0001$; effect of treatment $\left.F_{(1,36)}=11.07, p=0,0020\right)$. Post hoc analysis revealed significant differences in BMS score starting at 3 dpi and remaining signifi- 
A

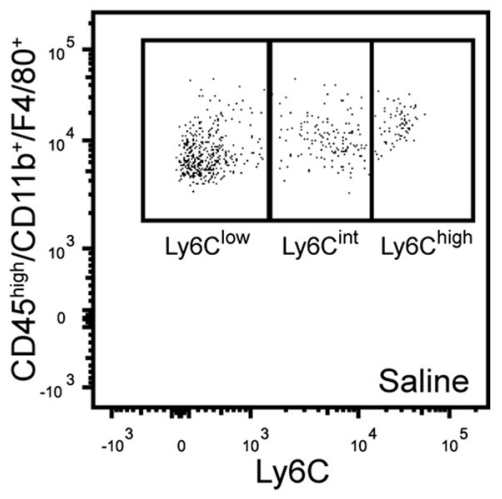

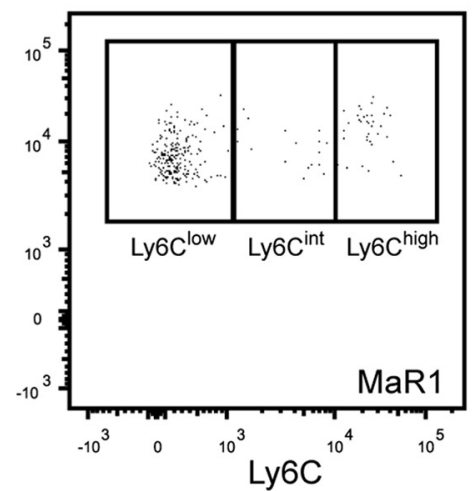

B

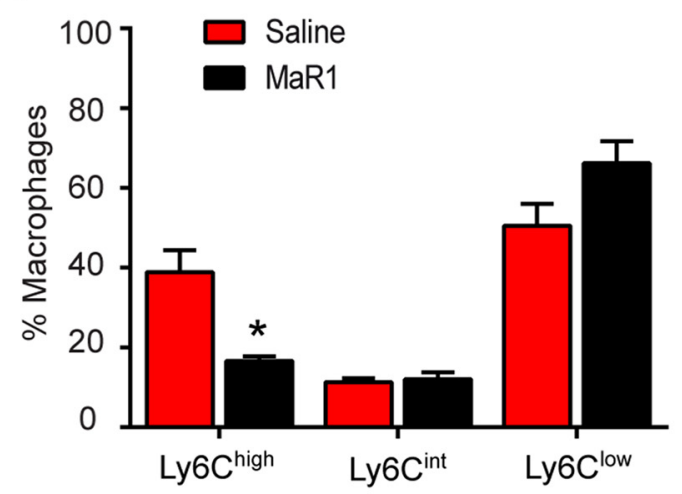

C
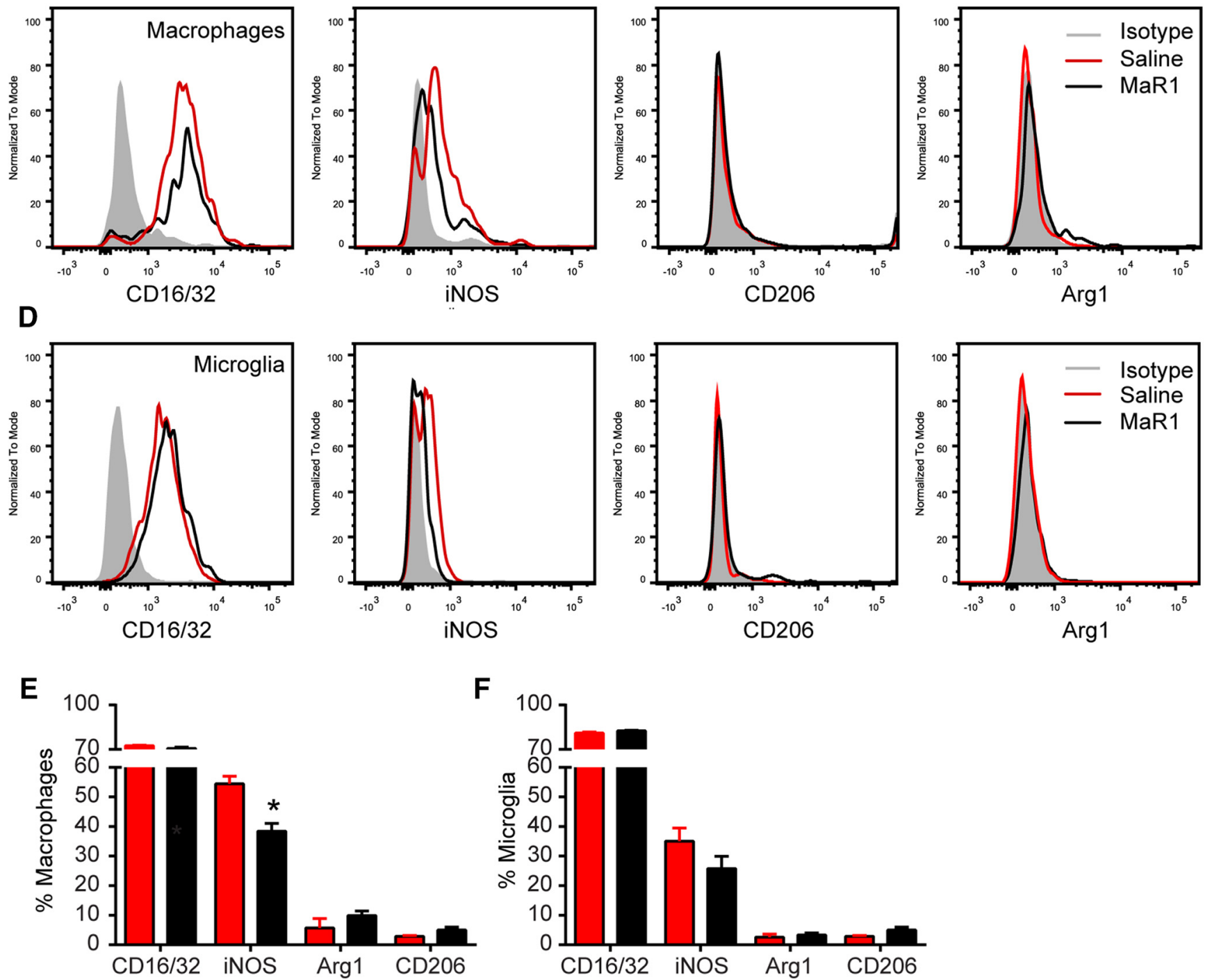

Figure 6. MaR1 redirects macrophages toward a prorepair phenotype after SCl. $\boldsymbol{A}$, Representative FACS analysis density plots of Ly6C macrophages in saline- and MaR1-treated mice at 7 dpi. $\boldsymbol{B}$, Graph showing proportion of different macrophage subsets in the injured spinal cord at $7 \mathrm{dpi}$. $\boldsymbol{C}, \boldsymbol{D}$, Representative FACS histograms plots of M1 and M2 markers in injured spinal cord for macrophages $\left(\boldsymbol{C}\right.$ ) and microglial cells $(\boldsymbol{D})$ at $7 \mathrm{dpi}$. $\boldsymbol{E}, \boldsymbol{F}$, Graphs showing the quantification of macrophages $(\boldsymbol{E})$ and microglial cells $(\boldsymbol{F})$ expressing $\mathrm{M} 1$ and $\mathrm{M} 2 \mathrm{markers}$ after $\boldsymbol{S C l}$. ${ }^{*} p<0.05$ versus saline. Student's $t$ test was used to analyze significant differences between MaR1 and control mice ( $n=6$ per group). Data are shown as mean \pm SEM.

cantly enhanced up the end of the follow-up (Fig. 8A). At $28 \mathrm{dpi}$, $50 \%$ of mice treated with saline showed plantar placement with no stepping, whereas the remaining $50 \%$ performed occasional stepping (BMS score of $3.5 \pm 0.22$ ). However, all mice treated with MaR1 displayed plantar placement with occasional or fre- quent stepping (score $4.58 \pm 0.22$ ). Mice administered Marl also showed faster speeds of locomotion on the treadmill, although this was not statistically significant (Mantel-Cox test $p=0.07$; Fig. $8 B$ ). In addition, DigiGait analysis revealed that MaR1 improved specific parameters of locomotion such as gait symmetry 
A

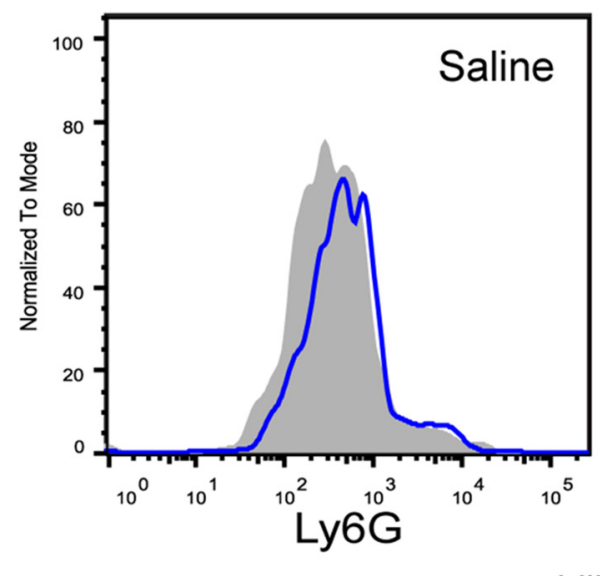

\section{non-permeabilized}

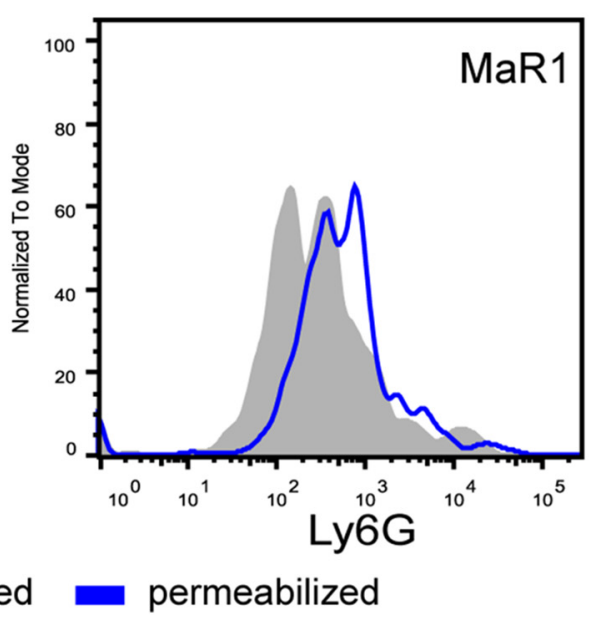

B

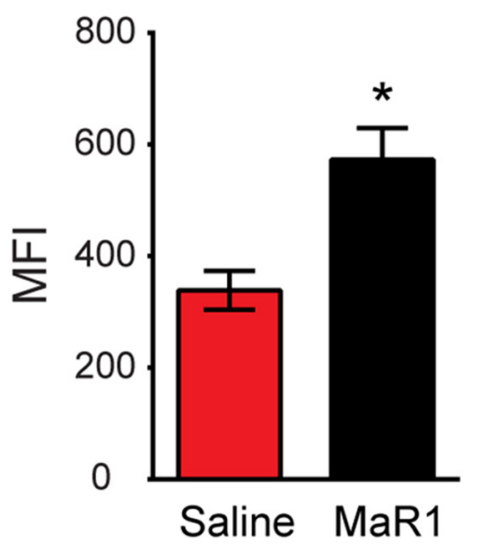

Figure 7. MaR1 increases efferocytosis after SCI. A, Representative FACS analysis histogram plots of the specific neutrophil marker Ly6G in macrophages at 7 dpi and after saline or MaR1 treatment. $\boldsymbol{B}$, Bar plot shows the increased in Ly6G median fluorescence intensity (MFI) in macrophages when the cell membrane is permeabilized, which is indicative of neutrophil phagocytosis. Note that MaR1 increased by $\sim 2$-fold the engulfment of neutrophils by macrophages. ${ }^{*} p<0.05$ versus saline. Mann-Whitney test was used to analyze significant differences ( $n=4$ per group). Data are shown as mean \pm SEM.

(Student's $t$ test, unpaired, two-tailed, $t=3.523 \mathrm{df}=7 ; p=$ 0.0097 ) and stance/width stepping variability (Student's $t$ test, unpaired, two-tailed, $t=2.465 \mathrm{df}=7 ; p=0.0431$; Fig. $8 C$ ) after SCI, further demonstrating improvement in locomotor control in mice treated with MaR1. No differences were found in other DigiGait parameters.

We then assessed whether the improvement in locomotor function of MaR1-treated mice was associated with a reduction in secondary tissue damage after SCI. Histological sections stained with LFB revealed that MaR1 increased myelin content at the injury epicenter and in sections located at $200 \mu \mathrm{m}$ rostral and caudal to the injury (Fig. $8 D, E$ ). To determine whether this greater amount of myelin was due to reduced demyelination, reduced axonal damage, or both, we quantified the number of axons $\left(\mathrm{NF}^{+}\right)$and those that had myelin sheath $\left(\mathrm{NF} / \mathrm{MBP}^{+}\right)$in the dorsal columns at the injury epicenter, the most damaged area of the spinal cord. These analyses reveal that MaR1 enhanced both axonal sparing (Student's $t$ test, unpaired, two-tailed, $t=$ $2.479 \mathrm{df}=18 ; p=0.0233$ ) and reduced demyelination after SCI (Student's $t$ test, unpaired, two-tailed, $t=2.931 \mathrm{df}=18 ; p=$ 0.0089; Fig. $8 F, G$ ). In addition, we found that MaR1 improved neuronal survival in the ventral horn in caudal regions to the injury epicenter (two-way ANOVA, Bonferroni's post hoc test; effect of day $F_{(16,306)}=64,43, p<0,0001$; effect of treatment $F_{(1,306)}=17,61 p<0,0001$; Fig. $\left.8 H, I\right)$. Overall, these data demonstrate that treatment with MaR1 reduces secondary tissue damage and improves functional outcomes after SCI.

\section{Discussion}

Traditionally, therapeutic approaches for acute SCI have sought to modulate the proinflammatory limb of the inflammatory response with limited success. Here, we identify impaired resolution of inflammation as a prominent feature of the dysregulated inflammatory response after SCI due to incomplete clearance of immune cells from the lesion site. We show that this impaired resolution coincides with severely blunted SPM biosynthesis, in contrast to peripheral, self-resolving inflammatory lesions, which are characterized by an early lipid mediator class shift (Serhan, 2014; Serhan et al., 2015). Our data reveal that systemic admin- istration of the resolution agonist MaR1 stimulated various biological mechanisms, which resulted in improved resolution of inflammation and marked improvement of locomotor outcomes.

PUFAs are key regulators of the inflammatory response because they control several processes involved in the onset and resolution of this physiological process (David et al., 2012c; Serhan, 2014; Serhan et al., 2015). Among them, n-3 PUFA ( $\omega 3$-fatty acids) has been specially brought to the attention of the scientific community due to its therapeutic effects in several inflammatory diseases. In particular, the n-3 PUFAs DHA and EPA, which are enriched in oils derived from fish and algae, are used extensively as dietary supplements and have been found to exert beneficial actions in a number of conditions in which inflammation contributes to the course of pathology, including SCI (King et al., 2006; Huang et al., 2007; López-Vales et al., 2010).

More recently, EPA and DHA lipid-derived mediators known collectively as SPMs have been identified as key players in the resolution of inflammation and regulators of homeostasis (Schwab et al., 2007; Buckley et al., 2014; Serhan, 2014; Serhan et al., 2015). The importance of SPMs in regulating inflammation is evident in many inflammatory disorders such as atherosclerosis, asthma, and ulcerative colitis, in which there is an absence of or insufficient or delayed production of SPMs (Serhan, 2014; Serhan et al., 2015). Importantly, the exogenous administration of SPMs reduces inflammation and prevents the detrimental effects exerted by the immune cells due to the failure to produce SPMs in the pathogenesis of different inflammatory diseases (Serhan, 2014; Serhan et al., 2015). Our results suggest that a similar scenario also occurs after SCI because the dysregulation of the resolution of inflammation coincides with the inefficient synthesis of SPMs.

Among the different family members of SPMs, maresins have been the least characterized. This family of SPMs derived from macrophages consists of two members, MaR1 (Serhan et al., 2009) and the more recently identified MaR2 (Deng et al., 2014). MaR1 exerts potent actions in regulating inflammation resolution, but also in preventing nociception after inflammatory- and chemotherapy-induced neuropathic pain and stimulating tissue 
A

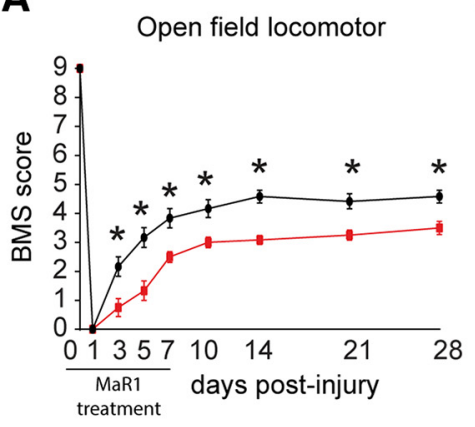

D

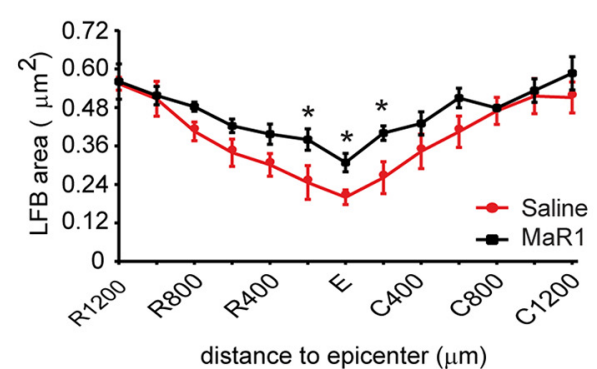

F
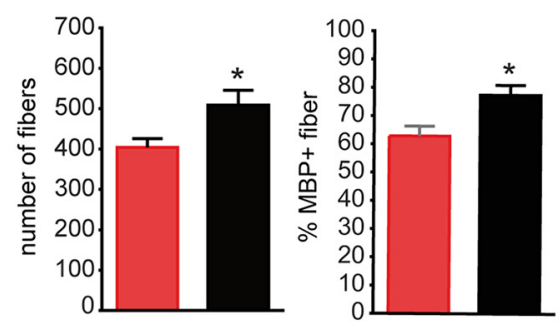

H

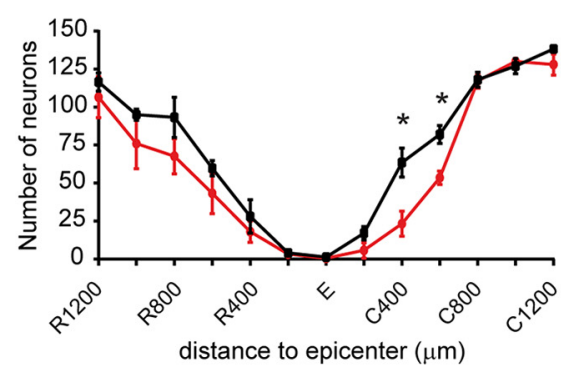

B

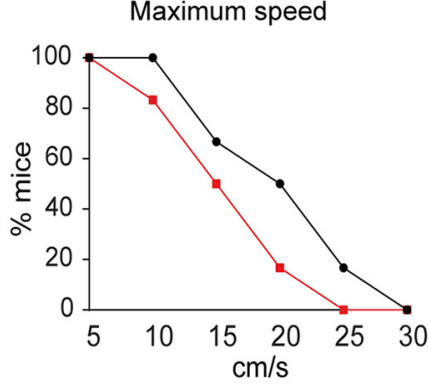

E

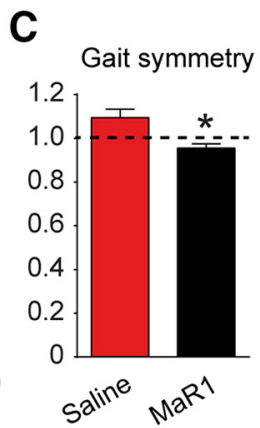

Stance/width

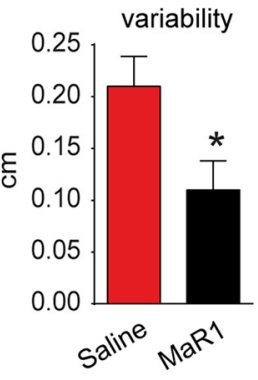

saline

MaR1
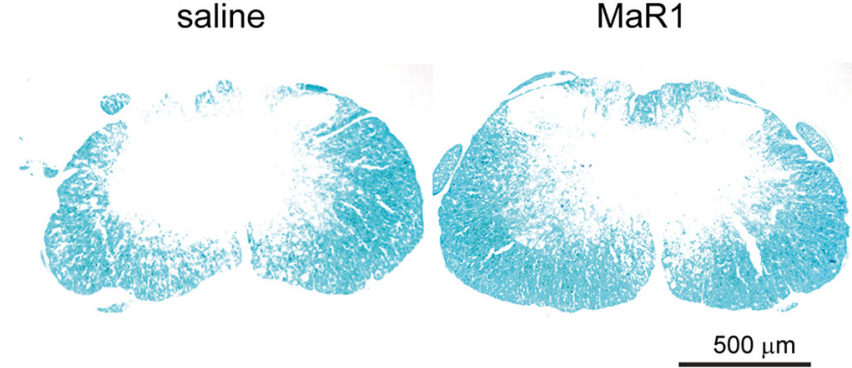

G
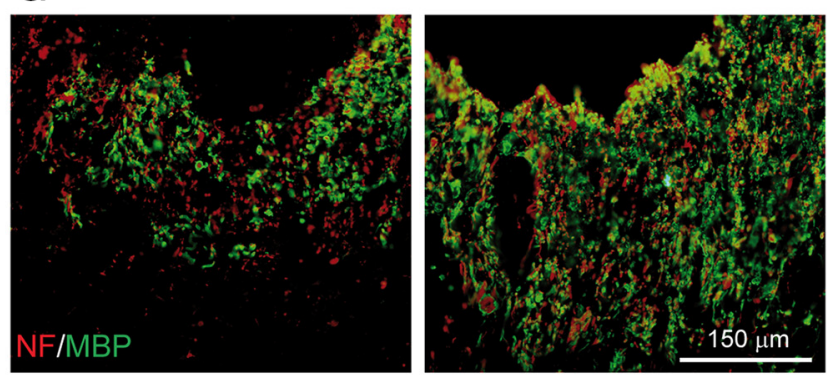

I
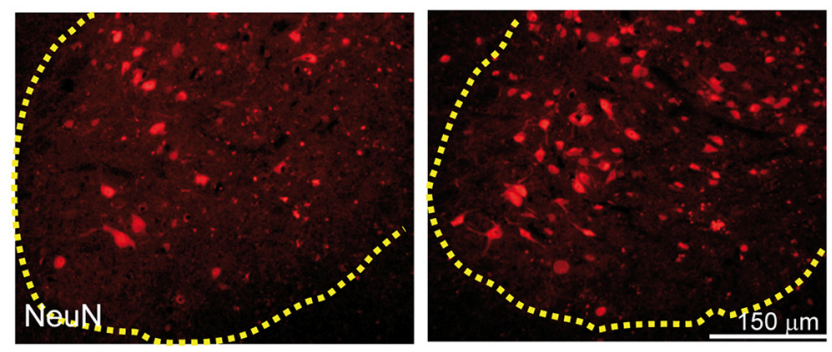

Figure 8. MaR1 improves locomotor recovery and attenuates secondary tissue damage after SCl. $\boldsymbol{A}$, Mice treated with MaR1 show significant improvement in locomotor skills assessed by the nine-point BMS. The BMS score of MaR1 treated mice inclined to significantly elevated levels starting at 3 dpi and remained consistent up the end of the follow-up (28 dpi) compared with saline-treated controls. $\boldsymbol{B}$, Mice administered MaR1 also showed faster speeds of locomotion on the treadmill. $\boldsymbol{C}$, DigiGait analysis revealed that MaR1 improved specific parameters of locomotion such as gait symmetry and stance/width stepping variability after SCl, further validating consolidated locomotor control in mice treated with MaR1. D, Quantification of myelin content at various distances rostral and caudal to the injury epicenter. $\boldsymbol{E}$, Representative micrographs showing myelin area at the injury epicenter in section stained with LFB from saline-treated (left) and MaR1-treated (right) mice. $\boldsymbol{F}$, Quantification of axons ( $\mathrm{NF}^{+}$) and myelinated axons ( $\mathrm{NF}^{+} / \mathrm{MBP}^{+}$) in the dorsal column at the injury epicenter from saline-treated (left) and MaR1-treated (right) mice. $\boldsymbol{G}$, Representative micrographs showing dorsal neurofilament (red) and MBP (green) staining at the injury epicenter from saline-treated (left) and MaR1-treated (right) mice. $\boldsymbol{H}$, Quantification of ventral horn neuron survival at various distances rostral and caudal to the injury epicenter revealing improved neuronal survival caudal to the lesion in the MaR1-treated group. $I$, Representative micrographs showing sparing of ventral horn neurons in saline-treated (left) and MaR1-treated (right) mice in tissue sections stained against NeuN at $400 \mu m$ caudal to the injury epicenter. ${ }^{*} p<$ 0.05; two-way repeated-measures ANOVA, Bonferroni's post hoc test in $\boldsymbol{A}, \boldsymbol{D}$, and $\boldsymbol{H}$; $t$ test in $\boldsymbol{C}$ and $\boldsymbol{F}$; Mantel-Cox test in $\boldsymbol{B}$ ( $n=10$ per group). Data are shown as mean \pm SEM.

regeneration in Planaria (Serhan et al., 2012; Serhan, 2014). Similar potent anti-nociceptive actions have also been demonstrated with other SPMs (Ji et al., 2011) and with some of the SPM pathway markers such as 17-HDHA (Lima-Garcia et al., 2011). Although the purpose of the present work was not to assess the effects of MaR1 on nociception after SCI, this should be considered for future studies because neuropathic pain compromises the quality of life in nearly $70 \%$ of SCI patients (Eaton, 2006). It should be noted that the resolving actions of MaR1 seem to be more potent than those exerted by other resolving agonists such 
as RvD1 because it was shown to stimulate greater efferocytosis by human macrophages at a $1 \mathrm{~nm}$ concentration (Serhan et al., 2012).

Here, we report that daily systemic treatment with very low doses of 7S-MaR1 (1 $\mu \mathrm{g} /$ mouse) after SCI accelerates and enhances neutrophil clearance and reduces macrophage accumulation in the lesioned spinal cord, two critical steps for the resolution of inflammation. 7S-MaR1 is an analog of MaR1 and is currently not known to been an isomer produced during biosynthesis. The present work shows that 7S-MarR1 shares the protective actions of MaR1 and can be useful for further studies on mechanisms of action of MaR1. Because this is, to the best of our knowledge, the first report assessing the effects of MaR1 in the CNS, we investigated the mechanisms underlying the resolving effects of MaR1 in SCI.

Recruitment of leukocytes into the lesioned spinal cord is regulated by proinflammatory mediators such as cytokines (David et al., 2012a; Popovich, 2014). MaR1 also downregulated the expression of cytokines in vitro in mouse models of both colitis and acute respiratory distress syndrome (Serhan et al., 2009; Marcon et al., 2013; Abdulnour et al., 2014). Our results in SCI indicate that MaR1 leads to reduced protein levels of several prominent proinflammatory cytokines in the spinal cord at $24 \mathrm{~h}$ after injury, including IL-6, CSF3, and different members of the chemokine family. Note also that MaR1 did not attenuate the expression of the anti-inflammatory cytokine IL10, suggesting a preferential action of this SPM in reducing proinflammatory cytokines.

Cytokines mediate inflammation by acting on specific receptors that activate different intracellular inflammatory cascades. Little is known about the intracellular cascades modulated by $\mathrm{MaR} 1$; however, a previous report showed that this resolving agent limited NF- $\kappa$ B activation (Marcon et al., 2013). Our results reveal that MaR1 did not abrogate this transcription factor after SCI. Similarly, PI3K/Akt signaling pathway was not affected by this SPM. MaR1 turned off several MAPK and JAK/STAT signaling pathways that are known to exert important proinflammatory actions in SCI without affecting the activation of STAT6, which is required for the suppressive effects of anti-inflammatory cytokines (David et al., 2012b). Both the cytokine and inflammatory signaling changes after MaR1 at $24 \mathrm{~h}$ after injury is likely to limit the subsequent infiltration of neutrophils and macrophages in the lesion site and, consequently, accelerate the reduction in their numbers after SCI.

Cytokines and signaling pathways also regulate the phenotype of macrophages. These cells can differentiate into two major types in vitro: (1) M1 macrophages, which display a proinflammatory profile and may mediate cytotoxic actions; and (2) M2 macrophages, which have anti-inflammatory effects and promote tissue healing and repair (Murray et al., 2014; David et al., 2015). However, microglia and macrophages in SCI cannot be defined within the simple M1-M2 dichotomy described in cell culture conditions, but rather form a broad spectrum of activation states (David et al., 2015; Francos-Quijorna et al., 2016). MaR1 was reported previously to shift the macrophage phenotype toward M2 in cell culture (Dalli et al., 2013). Here, we observed that, after SCI, MaR1 did not induce the expression of the classical M2 markers significantly in macrophages; however, it led to a significant reduction in the expression of M1 markers such as iNOS and Ly6C in macrophages, but not microglia. These results therefore suggest that MaR1 skews macrophage activation toward a phenotype more conducive for tissue repair in the lesioned CNS in vivo. Interestingly, this is not the only effect that $\mathrm{MaR} 1$ exerted on this leukocyte subset. We also found that the administration of this SPM stimulated macrophages to increase neutrophil phagocytosis in the lesioned spinal cord. Earlier studies have shown that MaR1 induced uptake of apoptotic neutrophils and $\mathrm{A} \beta_{42}$ in macrophages and microglia, respectively, in culture (Serhan et al., 2012; Zhu et al., 2016). However, this is the first study revealing that, similar to $\mathrm{RvD}$ and $\mathrm{RvE}, \mathrm{MaR} 1$ promotes neutrophil phagocytosis by macrophages in vivo (Schwab et al., 2007; Serhan, 2014). Therefore, the accelerated and increased clearance of neutrophils observed after SCI by MaR1 treatment could be explained by both the effects of this immunoresolvent agent on the phagocytic activity of macrophages to clear neutrophils (efferocytosis) and by its suppressive actions on cytokine levels and inflammatory signaling pathway activation. Recent studies demonstrated that MaR1 regulates $\mathrm{T}$ cells and platelets (Chiurchiù et al., 2016; Lannan et al., 2017), so we do not discard the idea that the beneficial actions of MaR1 in SCI could be also due to the effects of this SPMs on other cell types.

In conclusion, we provide clear evidence that MaR1 is effective at enhancing multiple stages of the resolution of inflammation after SCI. These include: downregulation of cytokines, silencing of inflammatory pathways, reduction of neutrophil and macrophage counts, shift in macrophage phenotype, and stimulation of the phagocytic activity of macrophages. Importantly, all of the biological effects induced by MaR1 treatment led to significant improvement in locomotor function and protection against secondary tissue damage. The present results support the concept that the inappropriate biosynthesis of SPMs after SCI hampers resolution of inflammation and contributes to the physiopathology of SCI. Because aberrant production of SPMs is also reported in the CSF of patients with Alzheimer's disease and multiple sclerosis (Prüss et al., 2013; Zhu et al., 2016), the administration of immunoresolvents may constitute an effective therapeutic avenue for the treatment of acute SCI and other neurological conditions in which inflammation contributes to the course of the disease and impaired function.

\section{References}

Abdulnour RE, Dalli J, Colby JK, Krishnamoorthy N, Timmons JY, Tan SH, Colas RA, Petasis NA, Serhan CN, Levy BD (2014) Maresin 1 biosynthesis during platelet-neutrophil interactions is organ-protective. Proc Natl Acad Sci U S A 111:16526-16531. CrossRef Medline

Arnold L, Henry A, Poron F, Baba-Amer Y, van Rooijen N, Plonquet A, Gherardi RK, Chazaud B (2007) Inflammatory monocytes recruited after skeletal muscle injury switch into antiinflammatory macrophages to support myogenesis. J Exp Med 204:1057-1069. CrossRef Medline

Basso DM, Fisher LC, Anderson AJ, Jakeman LB, McTigue DM, Popovich PG (2006) Basso Mouse Scale for locomotion detects differences in recovery after spinal cord injury in five common mouse strains. J Neurotrauma 23:635-659. CrossRef Medline

Buckley CD, Gilroy DW, Serhan CN (2014) Proresolving lipid mediators and mechanisms in the resolution of acute inflammation. Immunity 40: 315-327. CrossRef Medline

Chiurchiù V, Leuti A, Dalli J, Jacobsson A, Battistini L, Maccarrone M, Serhan CN (2016) Proresolving lipid mediators resolvin D1, resolvin D2, and maresin 1 are critical in modulating $\mathrm{T}$ cell responses. Sci Transl Med 8:353ra111. CrossRef Medline

Coll-Miró M, Francos-Quijorna I, Santos-Nogueira E, Torres-Espin A, Bufler P, Dinarello CA, López-Vales R (2016) Beneficial effects of IL-37 after spinal cord injury in mice. Proc Natl Acad Sci U S A 113:1411-1416. CrossRef Medline

Dalli J, Zhu M, Vlasenko NA, Deng B, Haeggström JZ, Petasis NA, Serhan CN (2013) The novel 13S,14S-epoxy-maresin is converted by human macrophages to maresin 1 (MaR1), inhibits leukotriene A4 hydrolase (LTA4H), and shifts macrophage phenotype. FASEB J 27:2573-2583. CrossRef Medline

David S, Kroner A (2011) Repertoire of microglial and macrophage responses after spinal cord injury. Nat Rev Neurosci 12:388-399. CrossRef Medline 
David S, López-Vales R, Wee Yong V (2012a) Harmful and beneficial effects of inflammation after spinal cord injury: potential therapeutic implications. Handb Clin Neurol 109:485-502. CrossRef Medline

David S, Zarruk JG, Ghasemlou N (2012b) Inflammatory pathways in spinal cord injury. Int Rev Neurobiol 106:127-152. CrossRef Medline

David S, Greenhalgh AD, López-Vales R (2012c) Role of phospholipase A2s and lipid mediators in secondary damage after spinal cord injury. Cell Tissue Res 349:249-267. CrossRef Medline

David S, Greenhalgh AD, Kroner A (2015) Macrophage and microglial plasticity in the injured spinal cord. Neuroscience 307:311-318. CrossRef Medline

Deng B, Wang CW, Arnardottir HH, Li Y, Cheng CY, Dalli J, Serhan CN (2014) Maresin biosynthesis and identification of maresin 2, a new antiinflammatory and pro-resolving mediator from human macrophages. PLoS One 9:e102362. CrossRef Medline

Eaton MJ (2006) Cell and molecular approaches to the attenuation of pain after spinal cord injury. J Neurotrauma 23:549-559. CrossRef Medline

Fawcett JW, Schwab ME, Montani L, Brazda N, Müller HW (2012) Defeating inhibition of regeneration by scar and myelin components. Handb Clin Neurol 109:503-522. CrossRef Medline

Francos-Quijorna I, Amo-Aparicio J, Martinez-Muriana A, López-Vales R (2016) IL-4 drives microglia and macrophages toward a phenotype conducive for tissue repair and functional recovery after spinal cord injury. Glia 64:2079-2092. CrossRef Medline

Gomez-Nicola D, Perry VH (2015) Microglial dynamics and role in the healthy and diseased brain: a paradigm of functional plasticity. Neuroscientist 21:169-184. CrossRef Medline

Hassan IR, Gronert K (2009) Acute changes in dietary omega-3 and omega- 6 polyunsaturated fatty acids have a pronounced impact on survival following ischemic renal injury and formation of renoprotective docosahexaenoic acid-derived protectin D1. J Immunol 182:3223-3232. CrossRef Medline

Hawthorne AL, Popovich PG (2011) Emerging concepts in myeloid cell biology after spinal cord injury. Neurotherapeutics 8:252-261. CrossRef Medline

Huang WL, King VR, Curran OE, Dyall SC, Ward RE, Lal N, Priestley JV, Michael-Titus AT (2007) A combination of intravenous and dietary docosahexaenoic acid significantly improves outcome after spinal cord injury. Brain 130:3004-3019. CrossRef Medline

Ji RR, Xu ZZ, Strichartz G, Serhan CN (2011) Emerging roles of resolvins in the resolution of inflammation and pain. Trends Neurosci 34:599-609. CrossRef Medline

King VR, Huang WL, Dyall SC, Curran OE, Priestley JV, Michael-Titus AT (2006) Omega-3 fatty acids improve recovery, whereas omega-6 fatty acids worsen outcome, after spinal cord injury in the adult rat. J Neurosci 26:4672-4680. CrossRef Medline

Kroner A, Greenhalgh AD, Zarruk JG, Passos Dos Santos R, Gaestel M, David S (2014) TNF and increased intracellular iron alter macrophage polarization to a detrimental M1 phenotype in the injured spinal cord. Neuron 83:1098-1116. CrossRef Medline

Lannan KL, Spinelli SL, Blumberg N, Phipps RP (2017) Maresin 1 induces a novel pro-resolving phenotype in human platelets. J Thromb Haemost 15:802-813. CrossRef Medline

Lima-Garcia JF, Dutra RC, da Silva K, Motta EM, Campos MM, Calixto JB (2011) The precursor of resolvin D series and aspirin-triggered resolvin D1 display anti-hyperalgesic properties in adjuvant-induced arthritis in rats. Br J Pharmacol 164:278-293. CrossRef Medline

López-Vales R, Redensek A, Skinner TA, Rathore KI, Ghasemlou N, Wojewodka G, DeSanctis J, Radzioch D, David S (2010) Fenretinide promotes functional recovery and tissue protection after spinal cord contusion injury in mice. J Neurosci 30:3220-3226. CrossRef Medline

Lu Y, Belin S, He Z (2014) Signaling regulations of neuronal regenerative ability. Curr Opin Neurobiol 27:135-142. CrossRef Medline
Marcheselli VL, Hong S, Lukiw WJ, Tian XH, Gronert K, Musto A, Hardy M, Gimenez JM, Chiang N, Serhan CN, Bazan NG (2003) Novel docosanoids inhibit brain ischemia-reperfusion-mediated leukocyte infiltration and pro-inflammatory gene expression. J Biol Chem 278:43807-43817. CrossRef Medline

Marcon R, Bento AF, Dutra RC, Bicca MA, Leite DF, Calixto JB (2013) Maresin 1, a proresolving lipid mediator derived from omega-3 polyunsaturated fatty acids, exerts protective actions in murine models of colitis. J Immunol 191:4288-4298. CrossRef Medline

Murray PJ, et al. (2014) Macrophage activation and polarization: nomenclature and experimental guidelines. Immunity 41:14-20. CrossRef Medline

Nahrendorf M, Swirski FK, Aikawa E, Stangenberg L, Wurdinger T, Figueiredo JL, Libby P, Weissleder R, Pittet MJ (2007) The healing myocardium sequentially mobilizes two monocyte subsets with divergent and complementary functions. J Exp Med 204:3037-3047. CrossRef Medline

Popovich PG (2014) Neuroimmunology of traumatic spinal cord injury: a brief history and overview. Exp Neurol 258:1-4. CrossRef Medline

Popovich PG, Longbrake EE (2008) Can the immune system be harnessed to repair the CNS? Nat Rev Neurosci 9:481-493. CrossRef Medline

Prüss H, Kopp MA, Brommer B, Gatzemeier N, Laginha I, Dirnagl U, Schwab JM (2011) Non-resolving aspects of acute inflammation after spinal cord injury (SCI): indices and resolution plateau. Brain Pathol 21:652660. CrossRef Medline

Prüss H, Rosche B, Sullivan AB, Brommer B, Wengert O, Gronert K, Schwab JM (2013) Proresolution lipid mediators in multiple sclerosis: differential, disease severity-dependent synthesis: a clinical pilot trial. PLoS One 8:e55859. CrossRef Medline

Santos-Nogueira E, López-Serrano C, Hernández J, Lago N, Astudillo AM, Balsinde J, Estivill-Torrús G, de Fonseca FR, Chun J, López-Vales R (2015) Activation of lysophosphatidic acid receptor type 1 contributes to pathophysiology of spinal cord injury. J Neurosci 35:10224-10235. CrossRef Medline

Schwab JM, Chiang N, Arita M, Serhan CN (2007) Resolvin E1 and protectin D1 activate inflammation-resolution programmes. Nature 447:869 874. CrossRef Medline

Serhan CN (2014) Pro-resolving lipid mediators are leads for resolution physiology. Nature 510:92-101. CrossRef Medline

Serhan CN, Yang R, Martinod K, Kasuga K, Pillai PS, Porter TF, Oh SF, Spite M (2009) Maresins: novel macrophage mediators with potent antiinflammatory and proresolving actions. J Exp Med 206:15-23. CrossRef Medline

Serhan CN, Dalli J, Karamnov S, Choi A, Park CK, Xu ZZ, Ji RR, Zhu M, Petasis NA (2012) Macrophage proresolving mediator maresin 1 stimulates tissue regeneration and controls pain. FASEB J 26:1755-1765. CrossRef Medline

Serhan CN, Dalli J, Colas RA, Winkler JW, Chiang N (2015) Protectins and maresins: New pro-resolving families of mediators in acute inflammation and resolution bioactive metabolome. Biochim Biophys Acta 1851:397413. CrossRef Medline

Steinman L (2015) No quiet surrender: molecular guardians in multiple sclerosis brain. J Clin Invest 125:1371-1378. CrossRef Medline

Stenudd M, Sabelström H, Frisén J (2015) Role of endogenous neural stem cells in spinal cord injury and repair. JAMA Neurol 72:235-237. CrossRef Medline

Svensson CI, Zattoni M, Serhan CN (2007) Lipoxins and aspirin-triggered lipoxin inhibit inflammatory pain processing. J Exp Med 204:245-252. CrossRef Medline

Zhu M, Wang X, Hjorth E, Colas RA, Schroeder L, Granholm AC, Serhan CN, Schultzberg M (2016) Pro-Resolving Lipid Mediators Improve Neuronal Survival and Increase Abeta42 Phagocytosis. Mol Neurobiol 53:27332749. CrossRef Medline 